\title{
A Computational and Experimental Study of Resonators in Three Dimensions
}

\author{
C.K.W. Tam ${ }^{1}$ and H. $\mathrm{Ju}^{2}$, \\ Florida State University, Tallahassee, FL 32306-4510 \\ M.G. Jones ${ }^{3}$, W.R. Watson ${ }^{4}$ and T.L. Parrott ${ }^{5}$ \\ NASA Langley Research Center, Hampton, VA 23681-2199
}

\begin{abstract}
In a previous work by the present authors, a computational and experimental investigation of the acoustic properties of two-dimensional slit resonators was carried out. The present paper reports the results of a study extending the previous work to three dimensions. This investigation has two basic objectives. The first is to validate the computed results from direct numerical simulations of the flow and acoustic fields of slit resonators in three dimensions by comparing with experimental measurements in a normal incidence impedance tube. The second objective is to study the flow physics of resonant liners responsible for sound wave dissipation. Extensive comparisons are provided between computed and measured acoustic liner properties with both discrete frequency and broadband sound sources. Good agreements are found over a wide range of frequencies and sound pressure levels. Direct numerical simulation confirms the previous finding in two dimensions that vortex shedding is the dominant dissipation mechanism at high sound pressure intensity. However, it is observed that the behavior of the shed vortices in three dimensions is quite different from those of two dimensions. In three dimensions, the shed vortices tend to evolve into ring (circular in plan form) vortices, even though the slit resonator opening from which the vortices are shed has an aspect ratio of 2.5 . Under the excitation of discrete frequency sound, the shed vortices align themselves into two regularly spaced vortex trains moving away from the resonator opening in opposite directions. This is different from the chaotic shedding of vortices found in two-dimensional simulations. The effect of slit aspect ratio at a fixed porosity is briefly studied. For the range of liners considered in this investigation, it is found that the absorption coefficient of a liner increases when the open area of the single slit is subdivided into multiple, smaller slits.
\end{abstract}

\section{Introduction}

Local-reacting acoustic liners are one of the most effective devices for suppressing fan noise from modern jet engines. For this reason, acoustic liners are presently installed in almost all commercial aircraft jet engines. Whenever surface area and space are available, engine designers tend to maximize the utilization of acoustic liners.

The structural simplicity of conventional liner structures (i.e., perforate over honeycomb core backed by impervious backing plate) belies the complexity of their functionality. This makes it difficult to accurately predict their acoustic properties. In the past, the impedance of a liner has been determined primarily via semi-empirical models and experiments. ${ }^{1-11}$ Fairly accurate predictions are possible via semi-empirical prediction models when used by experienced engineers considering a particular class of liners. However, when a significant change in the liner structure is made, the prediction of liner impedance becomes less reliable because of the semi-empirical basis of the prediction models. Recent advances in computational aeroacoustics (CAA) offer, for the first time, the potential of a first-principles prediction of acoustic liner properties. This is most desirable and attractive as the predicted liner

\footnotetext{
${ }^{1}$ Robert O. Lawton Distinguished Professor, Department of Mathematics. Fellow AIAA.

2 Research Associate, Department of Mathematics.

3 Senior Research Scientist, Structural Acoustics Branch, Research \& Technology Directorate. Associate Fellow AIAA.

${ }^{4}$ Senior Research Scientist, Computational Aerosciences Branch, Research \& Technology Directorate. Associate Fellow AIAA.

5 Senior Research Scientist, Structural Acoustics Branch, Research \& Technology Directorate.
} 
impedance would be free of empirical constants. In addition to the possibility of becoming an impedance prediction tool, CAA offers an enhancement to semi-empirical model prediction by better quantifying constants that heretofore have necessitated elaborate experimentation, thereby providing a means to investigate and better understand the mechanisms by which an acoustic liner dissipates incident sound waves. The openings of an acoustic liner are quite small. This makes it difficult to perform experimental observations and measurements of the fluid flow and acoustic fields in and around an acoustic liner. On the other hand, small liner openings are no hindrance to CAA simulations. Moreover, numerical simulations using CAA methodologies can provide a complete set of space-time data. This is an invaluable asset for flow physics investigation and analysis. At the present time, the goals of using CAA methodology for first-principles impedance prediction and for investigation of acoustic wave dissipation mechanisms have not been fully attained. Nevertheless, significant advances have been made. Further efforts are necessary to develop the technology into a prediction and a design code.

Recently, Tam and Kurbatskii ${ }^{12}$ initiated an investigation on liner physics and impedance prediction using direct numerical simulation (DNS). They recognized that the physics of the problem involved the interplay of the effects of viscosity and compressibility. Near the walls of the liner opening, the viscous effect is dominant and the viscous scale is small. A thin viscous Stokes layer forms adjacent to the wall. Very fine meshes are required to resolve this Stokes layer. Away from the walls, the dominant effect is compressibility, which gives rise to acoustic disturbances with long wavelengths relative to the slit width. In these regions, the use of a coarse mesh is sufficient. Tam and Kurbatskii took into account the scale difference of the problem and used the multi-size-mesh, multi-timestep, dispersion-relation-preserving (DRP) $s c h e m e^{13}$ in their numerical simulation. This numerical scheme allows the use of different size meshes in different regions of the computational domain. They also simplified their problem by considering a two-dimensional model.

Melling ${ }^{14}$ provides an excellent historical review of acoustically driven oscillatory flows in square edged orifices. Starting with Sivian ${ }^{17}$ in 1935, it has generally been known that such flows exhibit nonlinear acoustic resistance wherein the nonlinear increase in resistance is attributed to the irreversible conversion of acoustic energy into kinetic energy and/or turbulence. Of particular note is the seminal work of Ingard and Labate. ${ }^{9}$ Via stroboscopic observations, these researchers discovered four distinct flow regimes (regions in the particle-velocity-frequency plane) with parametric dependence upon orifice geometry. These regimes were described as follows (in order of increasing excitation level) for an orifice over a cavity, driven at resonance:

Regime 1: A low sound intensity regime with stationary circulation; flow directed out from orifice along axis.

Regime 2: A regime of stationary circulation in which the direction of flow along the axis is toward the orifice.

Regime 3: A medium sound intensity regime where pulsatory effects are superposed upon circulations of the kind in Regime 2.

Regime 4: A high sound intensity regime in which pulsatory effects are predominant, resulting in the formation of jets and vortex rings. The jet consists of strong air-flow through the orifice signified by a sudden burst of air. This burst appears symmetrically on both sides of the orifice and is made up of pulses contributed by each cycle of the sound wave.

It is clear that the quantitative results of the present investigation are consistent with the qualitative results attributed to Regime 4 as described by Ingard, et al. ${ }^{9}$ The current investigation (beginning with the work of Tam and Kurbatskii $^{12}$ ) is believed to represent the first quantification of these physical mechanisms via a first-principles CAA methodology. As such, this represents a significant advance for the understanding of oscillatory, orifice flows of interest in the predictive modeling of nonlinear acoustic resistance.

Experimental support for the vortex shedding dissipation mechanism is provided in the work of Tam, Kurbatskii, Ahuja and Gaeta. ${ }^{15}$ In their experiment, laser visualization was used to scan the unsteady flow field inside the resonator. Evidence of the observation of shed vortices was reported in their work. In addition, good agreement was found between absorption coefficients measured by normal incidence impedance tube experiment and direct numerical simulation. In spite of the good agreements found, experience with computational fluid dynamics (CFD) suggests that a more thorough validation of numerical simulation results is required. In an effort to provide further support of the validity of the results of direct numerical simulation of acoustic liners, a collaborative study was undertaken between the NASA Langley Research Center liner physics team and a Florida State University (FSU) team. Under this joint effort, the NASA team performed a series of normal incidence impedance experiments and measured the impedance of six specimens over the frequency range of 500 to $3000 \mathrm{~Hz}$ and SPLs of 120 to $155 \mathrm{~dB}$. The FSU team was responsible for conducting a corresponding series of virtual experiments using DNS. This being a first step in the validation process, two-dimensional (aspect ratio of 40) slit models were used. The extensive results of this joint study have been reported in Tam et al. ${ }^{16}$ Over the fairly large test parameter space, Tam et al showed that the computed liner impedance was in good agreement with experimental measurements. The 
good agreements lend strong support to the validity of the DNS approach, and provide verification that vortex shedding is the main dissipation mechanism of acoustic liners at high SPLs.
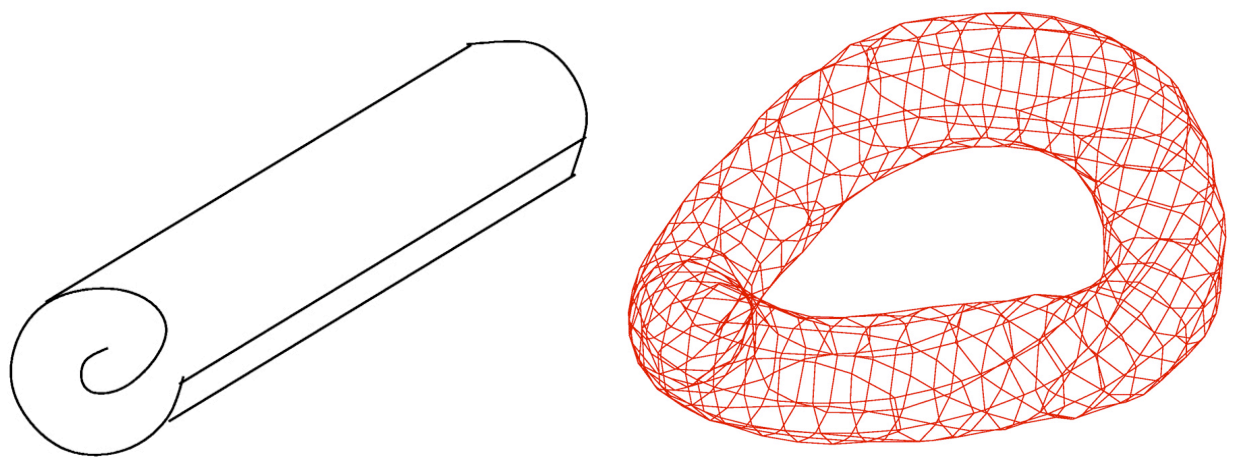

Figure 1. Sketch of a line vortex and a ring vortex

The present study is a continuation of the work reported by Tam et al. ${ }^{16}$ The primary objective is to provide three-dimensional validation tests for direct numerical simulation of the acoustic and flow fields inside a normal incidence impedance tube. Both discrete and broadband incident sound waves are considered. An improved method is introduced in this work. A secondary objective of this investigation is to clarify the behavior of the shed vortices. For slit resonators with large aspect ratios, the shed vortices are essentially line vortices as shown in Fig. 1. Previous work indicates that the movements of line vortices, once shed, are quite random and chaotic. However, for acoustic liners with an aspect ratio of the face sheet openings close to unity (i.e., slits shrinking toward squares), the shed vortices are in the form of ring vortices. Ring vortices have strong self and mutual interaction. It is our intention to observe their behavior using the DNS data and to report some of the salient features here.

The remainder of this paper is organized as follows. The experimental procedure and instrumentation are reported in section II. In section III, the three-dimensional computational model and computational algorithm are described. Treatment and analysis of simulation data for broadband incident sound waves are considered in Section IV. Numerical results and their comparisons with experimental measurements are reported in section V. Some interesting observations of vortex shedding and behavior of shed vortices are reported in Section VI. Section VII concludes this study.

\section{Experimental Instrumentation and Procedure}

In the present study, experimental data were acquired in a normal incidence impedance tube (NIT) located in the Flow Impedance Test Laboratory of the NASA Langley Research Center. A schematic diagram of the apparatus and associated electronics is shown in Fig. 2. The impedance tube has a square cross-section of 2 in by 2 in and a length of $24 \mathrm{in}$. A detailed description of the apparatus may be found in Ref.[16] and will, therefore, not be repeated here. The two-microphone method (TMM) of Jones and Parrott ${ }^{11}$ is implemented with two microphones flush-mounted 1.25 in apart in a rotating steel plate in the top wall. This allows the position of two 0.25 -in diameter condenser-type microphones to be switched in a very convenient and precise manner. The acoustic impedance of the test specimen, determined using the TMM, can then be used to separate the total acoustic pressure into incident and reflected acoustic pressures. 


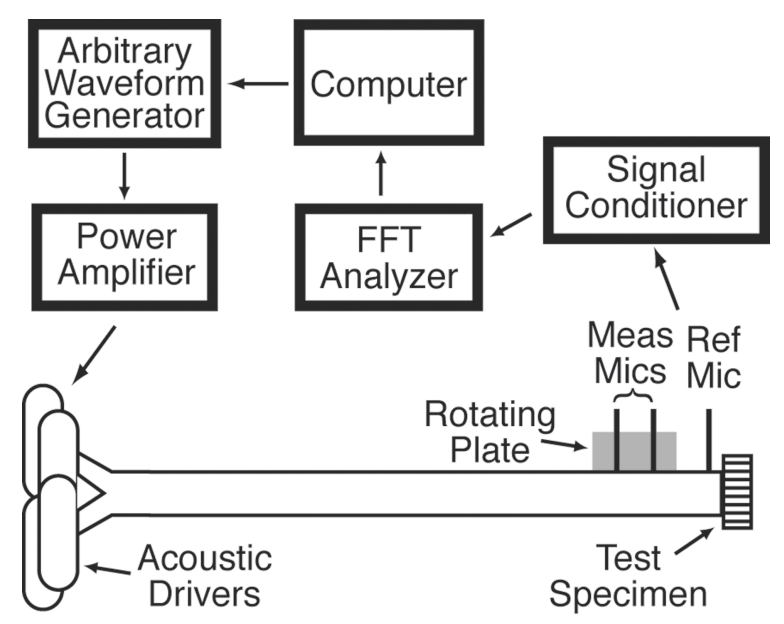

Figure 2. NASA LaRC normal incidence tube with instrumentation.

Six test specimens, all with the same open area, were manufactured. However, only data from three of the six specimens are used to compare with direct numerical simulation (DNS) measurements. We will refer to them as Samples 1, 5 and 6. The plan views of these specimens are shown in Fig. 3.

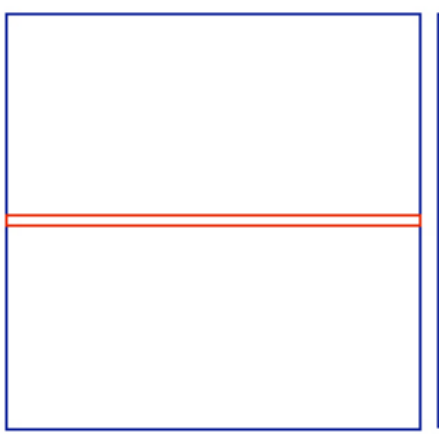

Sample 1

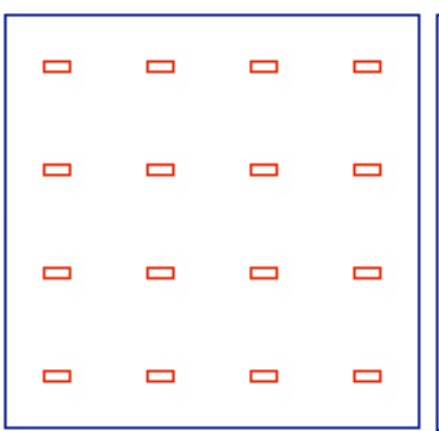

Sample 5

\begin{tabular}{|llll|}
\hline$\square$ & $\square$ & $\square$ & $\square$ \\
$\square$ & $\square$ & $\square$ & $\square$ \\
$\square$ & $\square$ & $\square$ & $\square$ \\
$\square$ & $\square$ & $\square$ & $\square$ \\
$\square$ & $\square$ & $\square$ & $\square$ \\
$\square$ & $\square$ & $\square$ & $\square$ \\
$\square$ & $\square$ & $\square$ & $\square$ \\
$\square$ & $\square$ & $\square$ & $\square$ \\
\hline
\end{tabular}

Sample 6

Figure 3. Slit configurations for test Samples 1, 5 and 6, with aspect ratios of 40, 2.5 and 1.25, respectively.

Sample 1 has a single 0.05 in by 2.0 in slit. Sample 5 has sixteen 0.05 in by 0.125 in slits. Sample 6 has thirty-two 0.05 in by 0.0625 in slits. Thus, the aspect ratios of the slits are 40, 2.5 and 1.25, respectively. The slit in Sample 1 is effectively two-dimensional. The data from this specimen has been used to compare with DNS measurements in Ref. [16]. For this reason, the present investigation concentrates on experimental data from the other two samples. With identical total open area, the three samples offer an opportunity to study the effect of hole size on liner impedance.

\section{Computational Model, Algorithm and Grid}

One of the principal objectives of this investigation is to perform direct numerical simulation of the normal incidence impedance tube experiments carried out at the NASA Langley Research Center. In this section, the computational model is presented. In order to have an accurate numerical simulation, a well-designed computational grid and a high resolution computational algorithm are required. The three-dimensional computational grid and time-marching algorithm used in this study are described below. 


\section{A. Mathematical Model}

The acoustic field inside a normal incidence impedance tube is governed by the compressible NavierStokes equations. With respect to the following scales [length $=D$ (thickness of resonator opening), velocity $=$ $a_{0}$ (speed of sound), time $=D / a_{0}$, density $=\rho_{0}$ (mean density of gas), pressure $=\rho_{0} a_{0}^{2}$, impedance $\left.=\rho_{0} a_{0}\right]$ the dimensionless Navier-Stokes equations are,

$$
\begin{aligned}
& \frac{\partial \rho}{\partial t}+\rho \frac{\partial u_{j}}{\partial x_{j}}+u_{j} \frac{\partial \rho}{\partial x_{j}}=0, \\
& \frac{\partial u_{i}}{\partial t}+u_{j} \frac{\partial u_{i}}{\partial x_{j}}=-\frac{1}{\rho} \frac{\partial p}{\partial x_{i}}+\frac{1}{\rho} \frac{\partial \tau_{i j}}{\partial x_{j}}, \\
& \frac{\partial p}{\partial t}+u_{j} \frac{\partial p}{\partial x_{j}}+\gamma p \frac{\partial u_{j}}{\partial x_{j}}=0,
\end{aligned}
$$

where

$$
\tau_{i j}=\frac{1}{R}\left(\frac{\partial u_{i}}{\partial x_{j}}+\frac{\partial u_{j}}{\partial x_{i}}\right)
$$

$R=\frac{D a_{0}}{v}$ is the Reynolds number and $\gamma$ is the ratio of specific heats. In this problem, there is no intrinsic characteristic velocity (among the input variables) other than the speed of sound. Thus, the Reynolds number, unlike standard fluid dynamics problems, is based on sound speed. Computationally, the choice of velocity scale makes little difference.

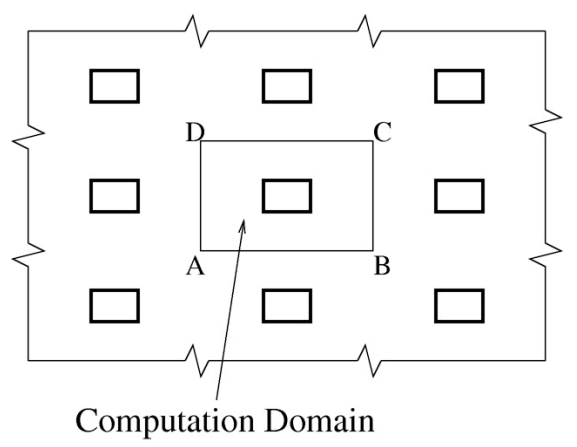

Figure 4. Computational domain for a one-slit model.

The Sample 5 and 6 specimens used in the NASA experiment have many openings in the face sheet (see Fig. 3). Computationally, it is not feasible to grid all the openings to reproduce the experimental condition precisely as this will require enormous number of mesh points and long computation time. To render the computation requirements reasonable, we will assume that the array of slits is infinite in extent. Under this condition, the acoustic field is spatially periodic. It is then sufficient to perform a computation for one slit with the imposition of periodic boundary conditions. Fig. 4 shows the plan view of the computational domain for a single slit. Periodic boundary conditions are imposed on vertical surfaces $\mathrm{AD}$ and $\mathrm{BC}$ as well as on vertical surfaces $\mathrm{AB}$ and $\mathrm{DC}$. The computational model in three dimensions is shown in Fig. 5. The dimensions are the same as in the NASA experiment. To ensure that the computed results of the single slit model are valid, a two-slit model with periodic boundary conditions as shown in Fig. 6 is also developed. It is found that the two-slit model gives essentially the same results as the one slit model. While this indicates the single-slit model is satisfactorily capturing the multi-slit phsyics, it also indicates the absence of interaction between adjacent holes. This is not altogether surprising, since the slit separation-to-slit diameter ratio is fairly large. This two-slit model offers the potential for exploration of this hole interaction effect as the hole separation is reduced. However, there was insufficient time to thoroughly investigate this effect in the current study. 


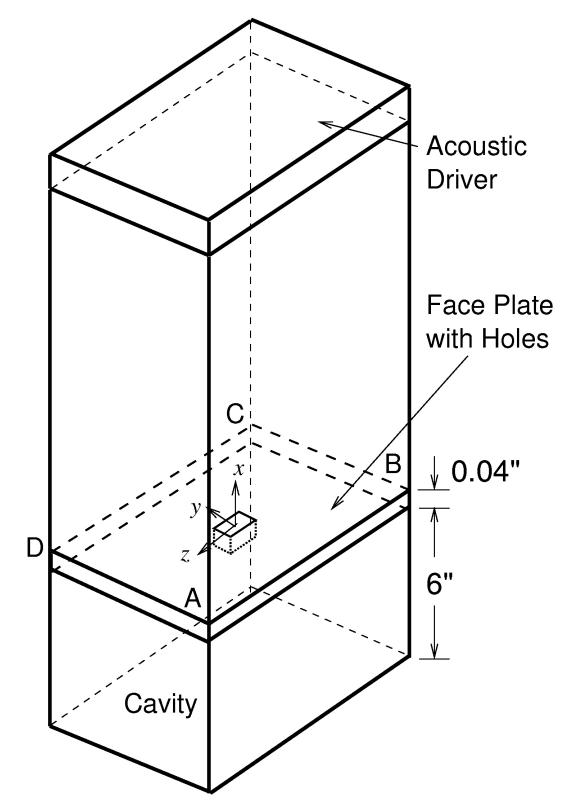

Figure 5. Three-dimensional, single-slit computational model.

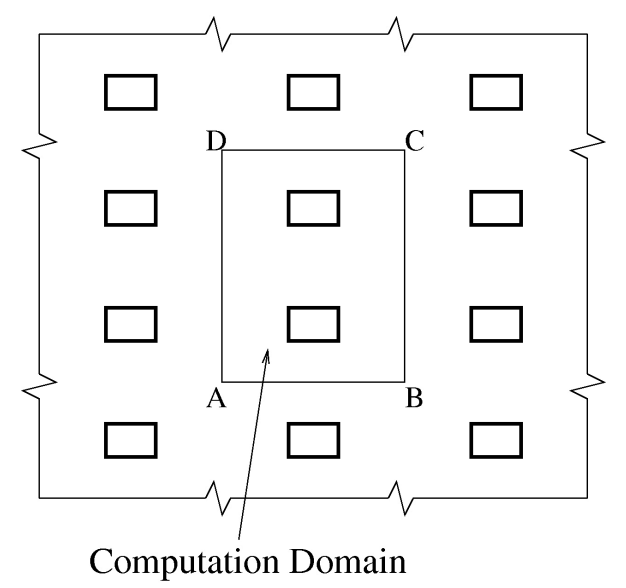

Figure 6. A two-slit computational model.

\section{B. Grid Design and Computational Algorithm}

It was pointed out in our previous work, Ref. [16], that the normal incidence impedance tube problem is a multi-scale problem. Near the walls of the slit, the viscous effect is dominant. The relevant length scale, L, is given by (see White ${ }^{18}$,

$$
L=2\left(\frac{\pi v}{f}\right)^{\frac{1}{2}}
$$

where $f$ is the frequency of oscillation. Away from the faceplate, the dominant effect on the dynamics of motion is compressibility. The length scale is the acoustic wavelength, $\lambda$, which is given by,

$$
\lambda=\frac{a_{0}}{f} \text {. }
$$

It is straightforward to show that $\lambda / L$ is equal to 320 for $6 \mathrm{kHz}$ sound at standard conditions. 
Because of the large disparity in length scales, a multi-size mesh is adopted. The time-marching computation is carried out using the multi-size-mesh, multi-time-step, dispersion-relation-preserving (DRP) scheme of Ref. [13]. The mesh sizes are chosen so that there are at least seven mesh points per wavelength. Seven mesh points per wavelength is the accuracy requirement of the 7-point stencil DRP scheme ${ }^{19}$. Fig. 7 shows the grid design in the $\mathrm{x}-\mathrm{z}$ plane for the Sample 6 computation. The smallest size mesh is in the sub-domain containing the slit opening. Here, the mesh size $\Delta$ is equal to $0.833 \times 10^{-3}$ in. The grid design in the $\mathrm{x}$-y plane (the smaller side of the slit) is shown in Fig. 8. It is to be noted that the mesh size in the sub-domain right at the slit opening is the same in the $\mathrm{x}, \mathrm{y}$, and z directions. In both Fig. 7 and 8 , the mesh size $\Delta_{k}$ means it is $k$ times that of $\Delta$, i.e. $\Delta_{k}=k \Delta$. The number preceding $\Delta_{k}$ is the number of mesh points of that size being used in that sub-domain.

For a viscous fluid, the boundary condition at a solid wall is the no-slip boundary condition. However, the viscous effect is unimportant away from the slit opening. To save computation time, the no-slip boundary condition is imposed only on the wall boundaries of the three sub-domains with the smallest size mesh. Effectively, the Euler equations are used for time-marching computation in the other sub-domains. The wall boundary conditions are enforced by the ghost point method as elaborated in Ref. [16].

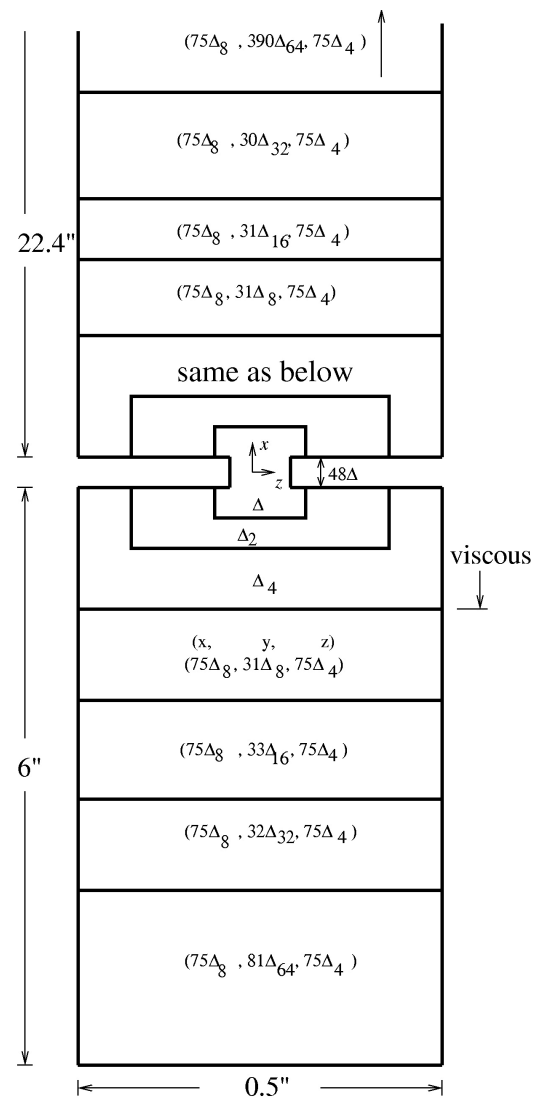

Figure 7. Mesh distribution in the $\mathrm{x}-\mathrm{z}$ plane.

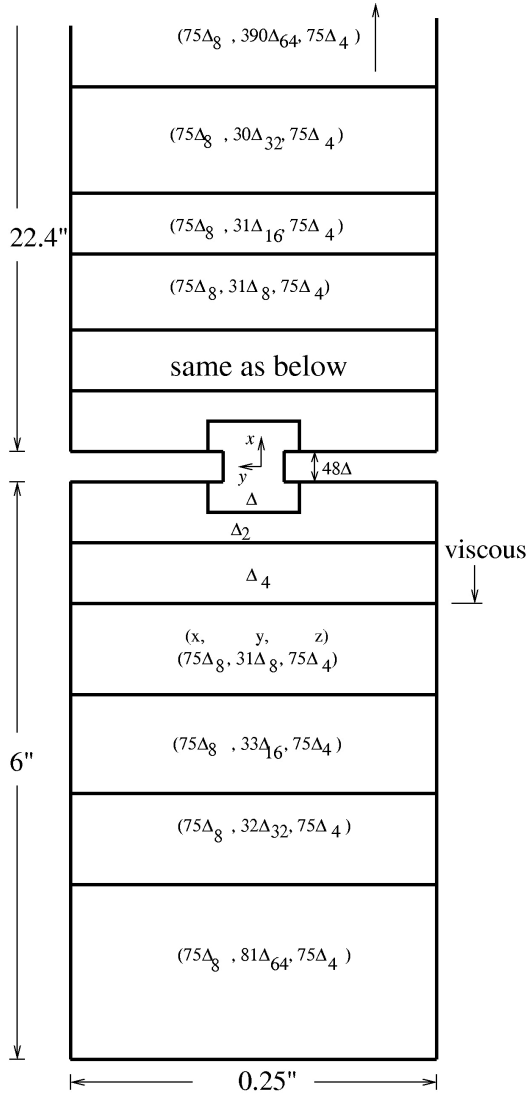

Figure 8. Mesh distribution in the x-y plane.

\section{Broadband Incident Acoustic Waves}

Acoustic liners are not only effective in suppressing tones. They can also be used to suppress broadband noise. One objective of the present investigation is to perform experimental measurements and direct numerical simulations with a broadband source. In our previous work on two-dimensional slit resonators, Ref. [16], broadband incident sound was considered. However, the accuracy of the proposed method for analyzing the simulation data was restricted to an incident sound field with a relatively narrow bandwidth. In this work, a much improved method is devised that has almost no bandwidth restriction. While this improved method allows modeling of broadband 
sound with a large bandwidth, it does not alleviate the need for sufficient sample length. It is well known that spurious oscillations can be introduced into the frequency spectrum if the input data sample is of insufficient duration. Due to CPU time restrictions, this problem persists.

\section{A. Energy Conserving Discretization}

The first crucial requirement in performing numerical simulation of broadband sound is the development of a method to computationally reproduce the experimentally measured noise spectrum. This is not a trivial problem. In our previous work, a way to discretize the input noise spectrum so as to reproduce the random noise signal in the time domain was described. This method of discretization is energy conserving. That is, the energy contained in a given frequency band of the noise spectrum in the computation is the same as that in the experimentally measured spectrum.

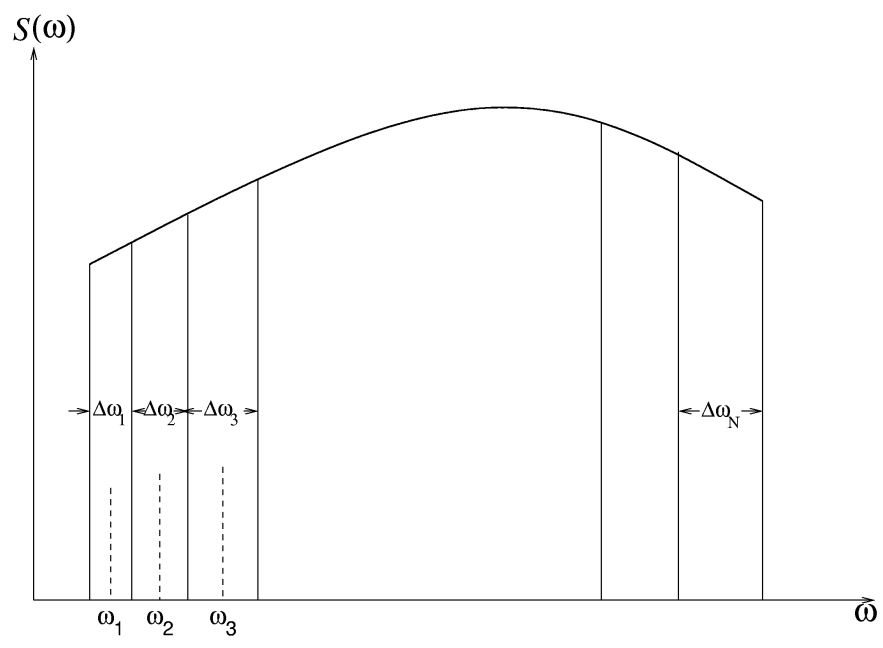

Figure 9. Energy conserving discretization of a given spectrum.

The energy conserving discretization method divides the measured noise spectrum into a large number of narrow bands with unequal bandwidth as show in Fig. 9. One important specification is that no center frequency of any frequency band is an integral multiple of the center frequency of another band. Harmonic interaction may be present in the measurement process due to the nonlinearity of the acoustic drivers. However, for the computational modeling, it is important that the discretization method avoid harmonic interaction, and the current scheme accomplishes this purpose. Suppose $\Delta \omega_{j}$ and $\omega_{j}$ are the bandwidth and center frequency $\left(\omega_{j}=2 \pi f_{j}\right.$ is the angular frequency) of the $j^{\text {th }}$ band, then the broadband sound field is mathematically represented by,

$$
p(t)=\sum_{j=1}^{N}\left(2 \Delta \omega_{j} S_{j}\right)^{\frac{1}{2}} \cos \left(\omega_{j} t+\chi_{j}\right)
$$

where $S_{j}$ is the spectrum level at $\omega_{j}$ and $\chi_{j}$ is a randomly assigned number. In the Appendix, it is shown that equation (7) is, indeed, an energy conserving discretization of a given spectrum $S(\omega)$ in the time domain. Fig. 10 is the time history of an input broadband sound field generated by equation (7). The spectrum is from one of the NASA Langley Research Center experiments during the present investigation. The time history of pressure fluctuations in Fig. 10 resembles those measured experimentally. 


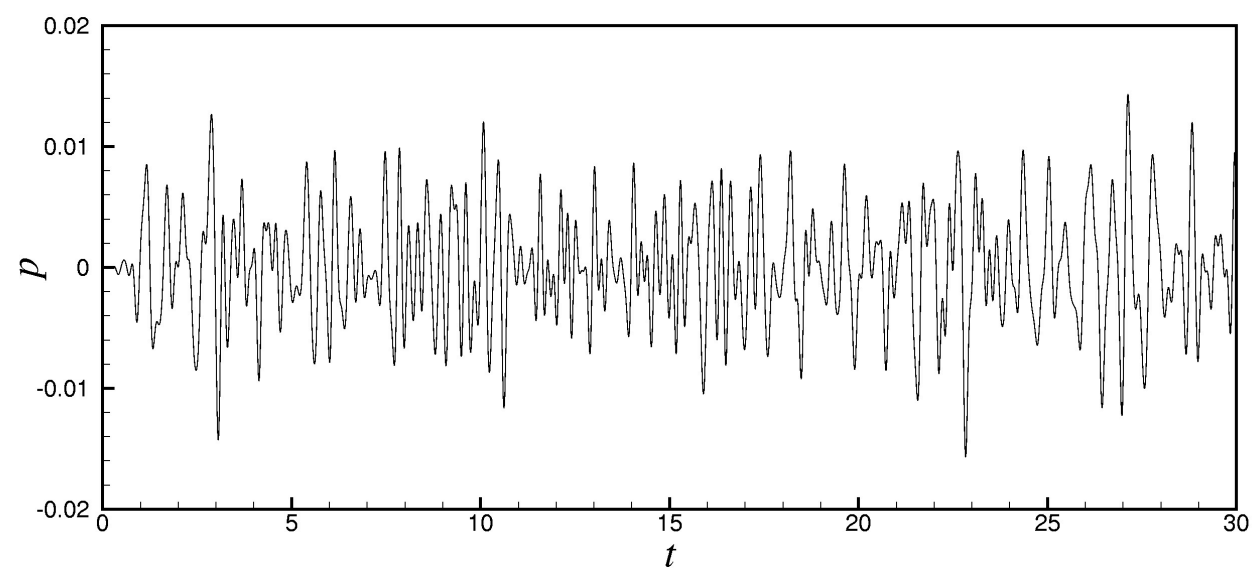

Figure 10. Time history of pressure fluctuations computed according to Eq. (7).

\section{B. Computation of Impedance and Absorption Coefficient}

The standard practice of measuring the normal incidence acoustic impedance (Note: all impedances used herein are assumed to be normalized by the characteristic impedance of air, $\rho_{0} a_{0}$ ) of a liner specimen in a normal incidence impedance tube is to use a two-microphone method as shown in Fig. 11. In our previous work, Ref. [16], a way to analyze the output from the simulated microphone signals for determining the impedance of the line specimen was discussed. However, it was noted that the method was subjected to certain limitations. As a part of the present investigation, an improved method has been developed. This method is described below.

It is assumed that only plane waves are present in the normal incidence impedance tube away from the surface of the liner. Without loss of generality, the pressure and velocity fields inside the tube may be written in the form,

$$
\begin{aligned}
& p(x, t)=\int_{-\infty}^{\infty}\left[A(\omega) e^{i \omega x}+B(\omega) e^{-i \omega x}\right] e^{-i \omega t} d \omega, \\
& u(x, t)=\int_{-\infty}^{\infty}\left[A(\omega) e^{i \omega x}-B(\omega) e^{-i \omega x}\right] e^{-i \omega t} d \omega,
\end{aligned}
$$

where $A(\omega)$ and $B(\omega)$ are the amplitude functions of the reflected and incident waves. Let $Z(\omega)$ be the impedance of the resonator. The impedance boundary condition at $x=0$ is,

$$
\tilde{p}(\omega)=-Z(\omega) \tilde{u}(\omega),
$$

where $\sim$ indicates Fourier transform and an $e^{-i \omega t}$ time dependence is assumed. By definition, $\tilde{p}(\omega)$ and $\tilde{u}(\omega)$ are equal to the quantities inside the square brackets of Eqs. (8) and (9). Upon substitution into Eq. (10), it is easy to find,

$$
Z(\omega)=\frac{A(\omega)+B(\omega)}{B(\omega)-A(\omega)}
$$




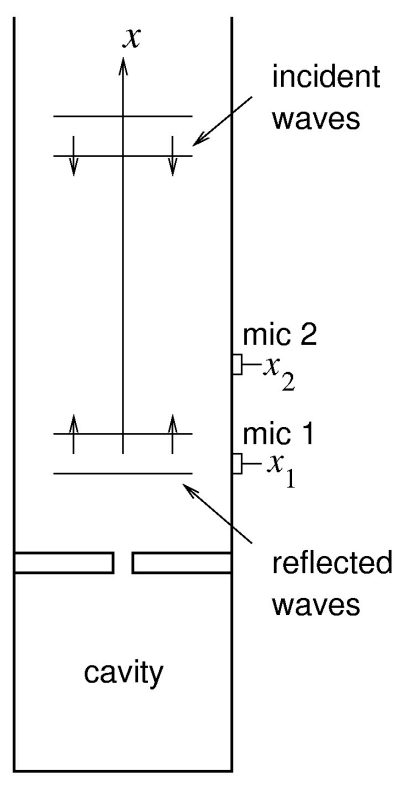

Figure 11. Schematic diagram of a normal incidence impedance tube showing incident and reflected plane waves and the locations of microphone 1 and 2.

Now, let $T_{1}(t)$ be the output of microphone 1 at $x_{1}$ and $T_{2}(t)$ be that of microphone 2 at $x_{2}$ (see Fig. 11). The Fourier transforms of these outputs are,

$$
\tilde{T}_{1}(\omega)=\frac{1}{2 \pi} \int_{-\infty}^{\infty} T_{1}(t) e^{i \omega t} d t ; \quad \tilde{T}_{2}(\omega)=\frac{1}{2 \pi} \int_{-\infty}^{\infty} T_{2}(t) e^{i \omega t} d t
$$

But $T_{1}(t)$ and $T_{2}(t)$ are given by Eq. (8) with $x=x_{1}$ and $x_{2}$ respectively. It follows that

$$
\begin{aligned}
& \tilde{T}_{1}(\omega)=A(\omega) e^{i \omega x_{1}}+B(\omega) e^{-i \omega x_{1}} \\
& \tilde{T}_{2}(\omega)=A(\omega) e^{i \omega x_{2}}+B(\omega) e^{-i \omega x_{2}} .
\end{aligned}
$$

It is straightforward to solve Eqs. (13) and (14) for $A(\omega)$ and $B(\omega)$. Upon substitution into Eq. (11), it is easy to obtain,

$$
Z(\omega)=i\left[\frac{\tilde{T}_{1}(\omega) \sin \left(\omega x_{2}\right)-\tilde{T}_{2} \sin \left(\omega x_{1}\right)}{\tilde{T}_{1}(\omega) \cos \left(\omega x_{2}\right)-\tilde{T}_{2} \cos \left(\omega x_{1}\right)}\right]
$$

Once the normalized normal incidence impedance $Z(\omega)$ is found by Eq. (15), the corresponding normalized reflection factor and absorption coefficient may be found by

$$
\text { Ref Factor }=\frac{Z(\omega)-1}{Z(\omega)+1}, \quad \text { Absorption Coefficient }=1-\left|\frac{Z(\omega)-1.0}{Z(\omega)+1.0}\right|^{2} .
$$




\section{Numerical Results and Comparisons with Experiments}

For this investigation, we concentrate our effort on experimental validation of DNS results for Test Samples 5 and 6. (Results of the validation tests for Sample 1 were previously reported in Ref. [16].) Two types of tests are performed in the present study - one with discrete frequency incident sound and the other with broadband acoustic waves. Since the response of a resonator to discrete frequency sound is quite different from that of broadband noise, the results are reported separately below.

\section{A. Discrete Frequency Incident Sound}

\section{Sample 6 (32 slits) results}

Two test series were performed with Sample 6, in which the source frequency was held constant and the incident sound pressure level (SPL) was varied. The first series was conducted at $2000 \mathrm{~Hz}$, and the second was conducted at $2500 \mathrm{~Hz}$. These frequencies were chosen for potential comparison with earlier results based on twodimensional DNS computations.

For the test series conducted at $2000 \mathrm{~Hz}$, the incident sound pressure level (SPL) was varied from $114 \mathrm{~dB}$ to $145 \mathrm{~dB}$. This variation in SPL level is $31 \mathrm{~dB}$, which is quite substantial. Figs. 12 and 13 show the measured and computed liner impedance (resistance and reactance) as a function of incident sound pressure level. In all the simulations, the pressure output of a pair of microphones located at 2.5 and 3.5 in from the surface of the resonator are used for calculating the impedance. These are the same as the locations of the microphones used in the experiment. To provide a direct check on the accuracy of the simulation, the measured signals of a second pair of microphones located at 10 and 11 in from the surface of the resonator are also used. We have found in all the test cases that the two pairs of microphones give virtually identical resonator impedance. Hence, only the results for the pair of microphones located at 2.5 and 3.5 in from the resonator surface are shown. The measured normalized resistance is nearly 0.35 (recall that impedances in this paper are normalized by $\rho_{0} a_{0}$ ) below the computed value at the lowest incident SPL, but this separation is reduced as the incident SPL is increased. The measured and computed reactance differences are larger, with a consistent difference of nearly 0.8 between the two sets of results over the entire range of incident SPLs.

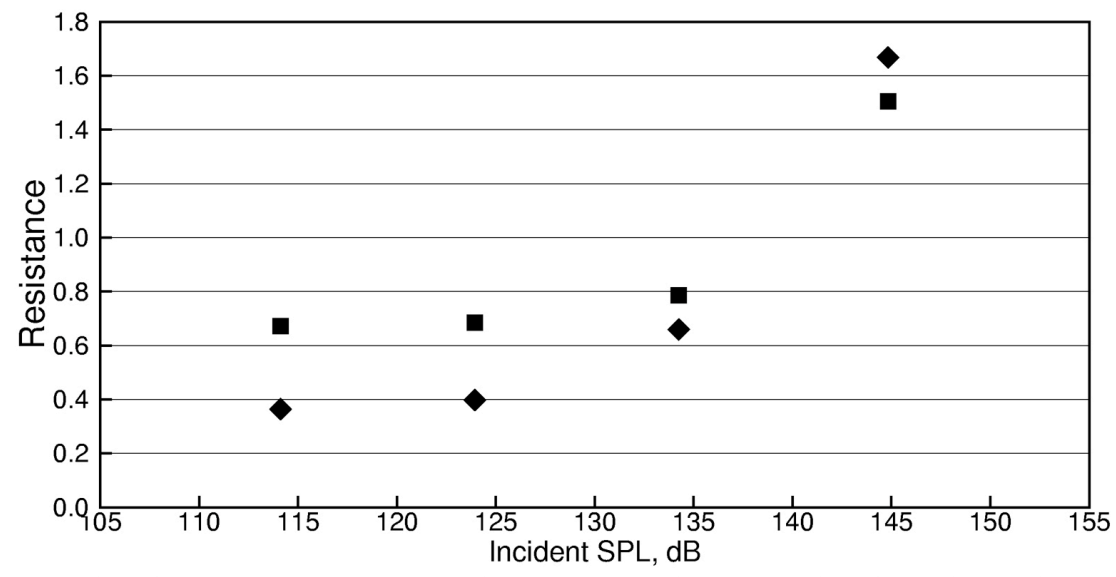

Figure 12. Normalized acoustic resistance at different sound pressure levels with 32-slit sample; incident sound frequency of $2 \mathrm{kHz}$. $\diamond$, experiment; $\mathbf{\square}$, simulation. 


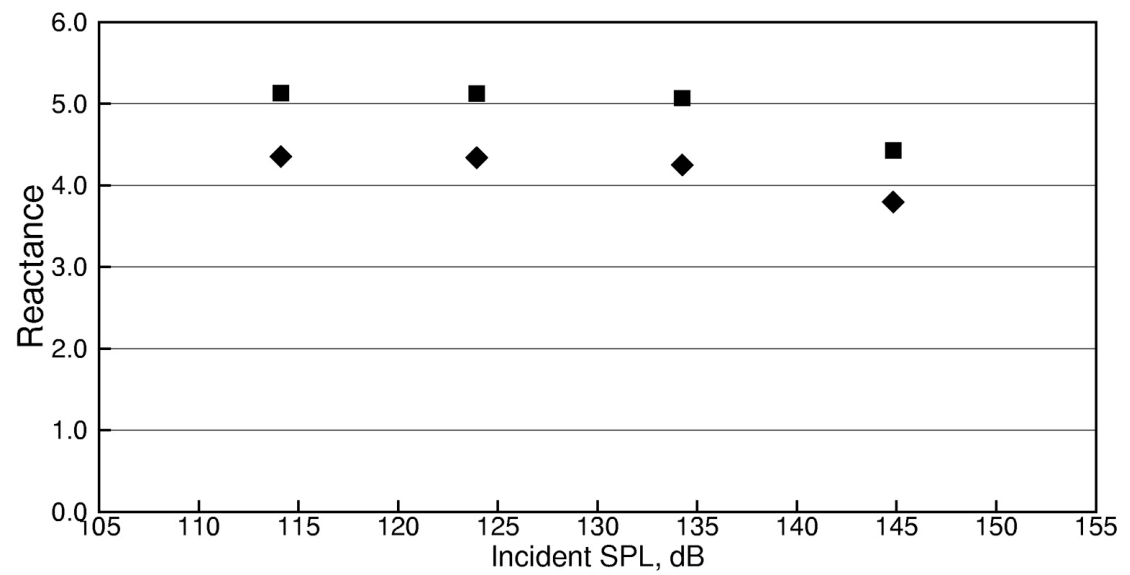

Figure 13. Normalized acoustic reactance at different sound pressure levels with 32-slit sample; incident sound frequency of $2 \mathrm{kHz}$. $\diamond$, experiment; $\mathbf{a}$, simulation.

These impedance differences between the DNS and experimental results are clearly of concern. However, it should be noted that cavity anti-resonances occur at 1125 and $2250 \mathrm{~Hz}$. When combined with the mass reactance of the liner, this will result in anti-resonant frequencies for the entire resonator (facesheet plus cavity) that are below those of the cavity alone. Hence, the choice of $2000 \mathrm{~Hz}$ is problematic, and represents one of the more difficult cases for which to make these comparisons. Nevertheless, it provides a worthy goal to demonstrate eventual success.

A better understanding of these differences emerges when the data are replotted in a different format. Figs. 14 and 15 provide the corresponding measured and computed normalized reflection factor (magnitude and phase) spectra. Figure 14 shows the reflection factor magnitude to be well matched for all but the highest SPL, with only a slight degradation in the comparison at this SPL. Figure 15 shows the measured reflection factor phase to be consistently higher than that computed via DNS. The difference is approximately 4 degrees for all but the highest SPL, and decreases to 2 degrees at the highest SPL. Hence, at least for this test condition, it appears that the differences in the measured and computed impedances are likely the result of a problem associated with the phase.

There are at least three possible causes for this phase difference. First, the DNS simulations were all conducted at a constant sound speed of $340 \mathrm{~m} / \mathrm{s}$ (assumed standard day conditions), whereas the sound speed in the experiments was approximately $345 \mathrm{~m} / \mathrm{s}$. This difference in sound speeds will cause a constant phase shift between the simulation and the measurement, and is of the appropriate magnitude to result in the phase shift observed in figure 15. However, as will be shown for other test conditions, the phase difference is not always constant. Thus, it would appear that this difference in sound speeds is not the sole cause of differences between the simulations and measurements. Regardless, this effect should be included in future computations. Second, measurement of incident SPL at the surface of the resonator requires accurate measurements, especially for frequencies near anti-resonance. Thus, it may be necessary to revisit the experiment with more detailed measurements. Finally, the computational domain selected for the DNS simulations may cause this phase difference. These simulations do not account for the effects of the walls of the normal incidence tube, as they are currently assumed to be sufficiently far from the resonator opening such that they can be neglected. However, near the surface of a nonlinear liner, higher-order modes are set up between the opposite walls of the normal incidence tube. These higher-order modes die out before they get to the location of the microphones, but they influence the near-field effect of the resonator openings. It is therefore possible that neglecting this effect may result in a phase difference in the results computed at the microphone locations. Each of these possibilities should be explored in future investigations. 


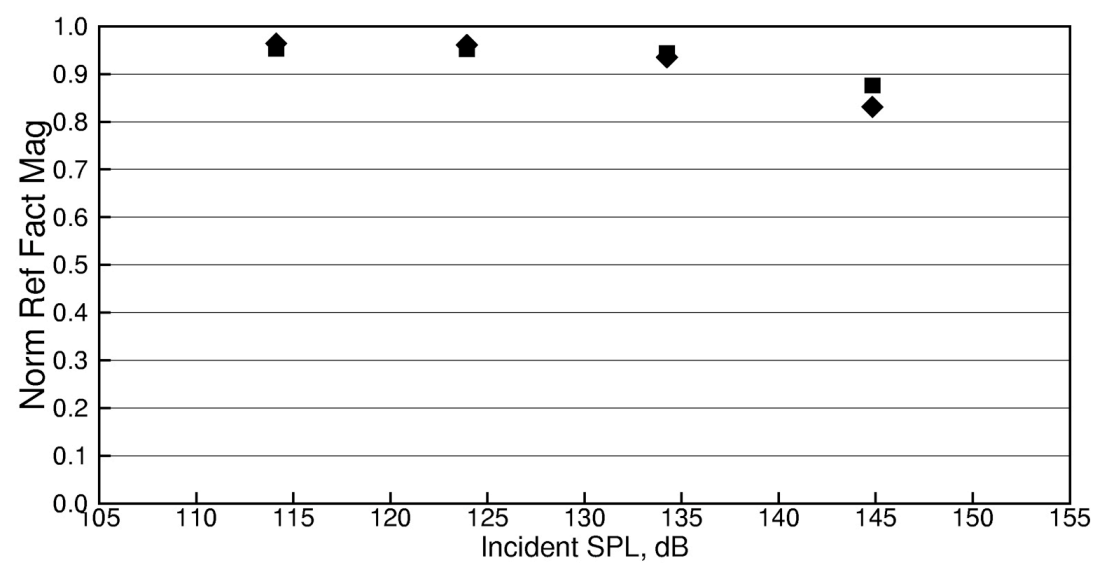

Figure 14. Normalized reflection factor magnitude at different sound pressure levels with 32-slit sample; incident sound frequency of $2 \mathrm{kHz}$. $\diamond$, experiment; $\mathbf{\square}$, simulation.

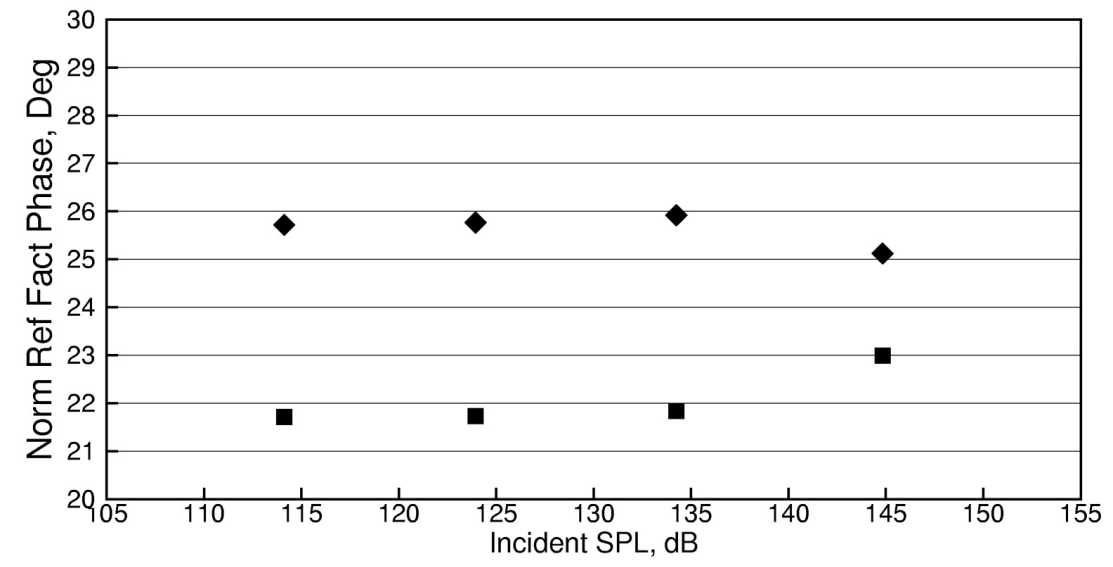

Figure 15. Normalized reflection factor phase at different sound pressure levels with 32-slit sample; incident sound frequency of $2 \mathrm{kHz}$. $\diamond$, experiment; $\mathbf{\square}$, simulation.

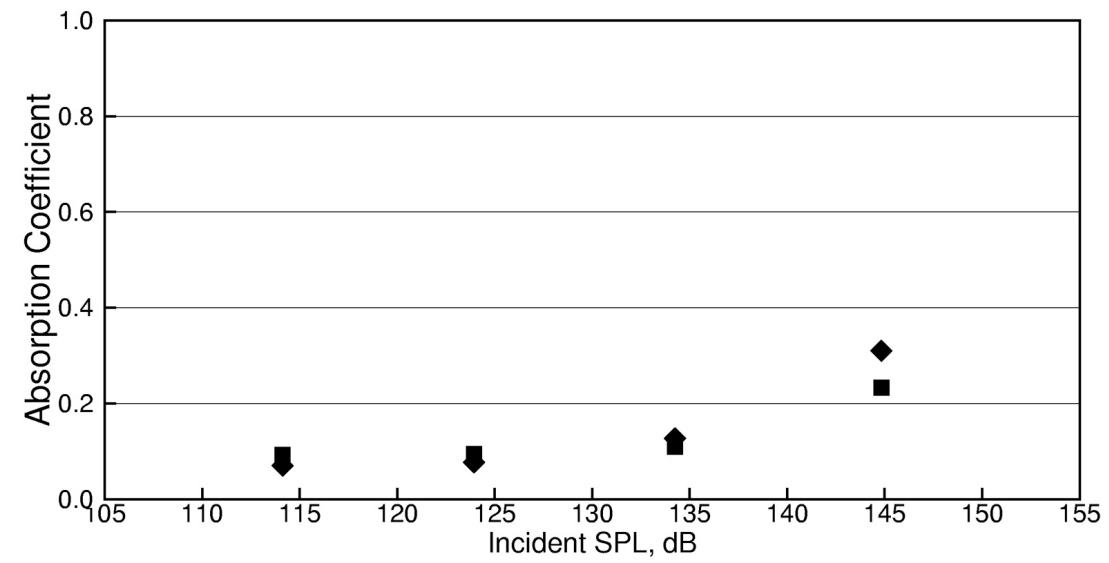

Figure 16. Absorption coefficient at different sound pressure levels with 32-slit sample; incident sound frequency of $2 \mathrm{kHz}$. $\diamond$, experiment; $\mathbf{\square}$, simulation.

Figure 16 provides the corresponding absorption coefficient spectra. Although the absorption is quite low, there is quite good agreement between the measured and computed results. Thus, for a normal incidence tube environment, the differences in the measured and computed impedances are not significant. However, the reader is reminded that the absorption coefficient is not an intrinsic parameter of the liner, and instead only provides 
information regarding how the liner will respond in the selected environment. Thus, since we wish to eventually use the DNS approach to model liners used in actual aircraft engine environments, comparisons of the measured and computed liner impedances (or alternatively, the reflection factor) remain as the appropriate figure of merit.

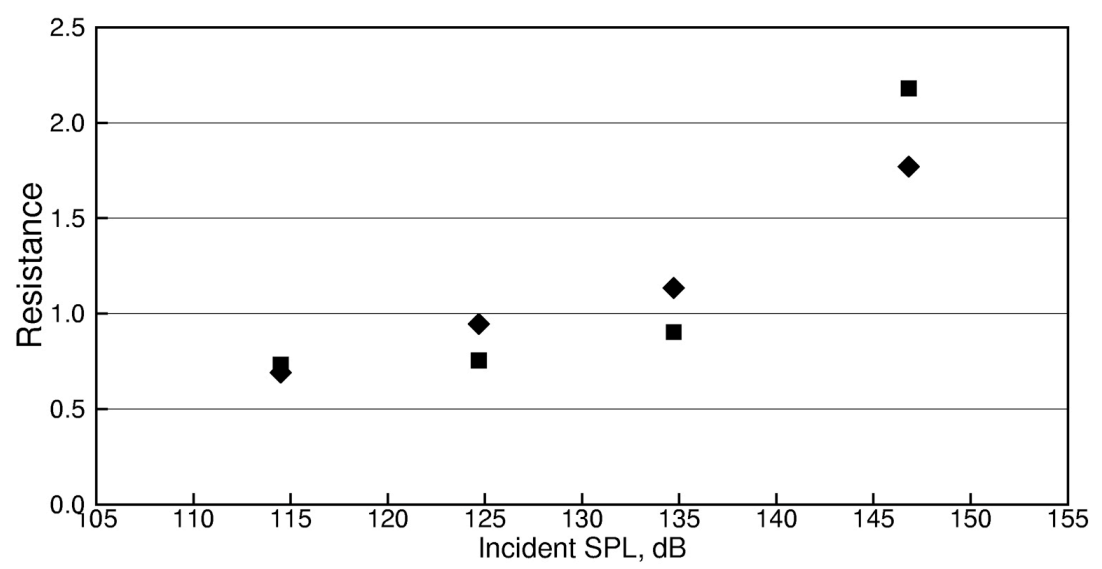

Figure 17. Normalized acoustic resistance at different sound pressure levels with 32-slit sample; incident sound frequency of $2.5 \mathrm{kHz}$. $\diamond$, experiment; $\mathbf{\square}$, simulation.

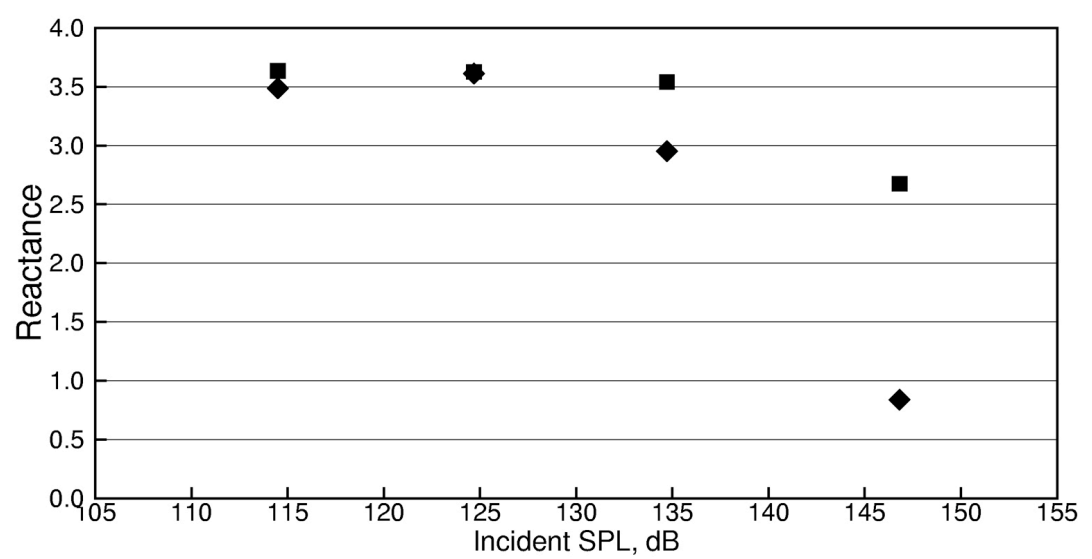

Figure 18. Normalized acoustic reactance at different sound pressure levels with 32-slit sample; incident sound frequency of $2.5 \mathrm{kHz}$. $\diamond$, experiment; $\mathbf{\square}$, simulation.

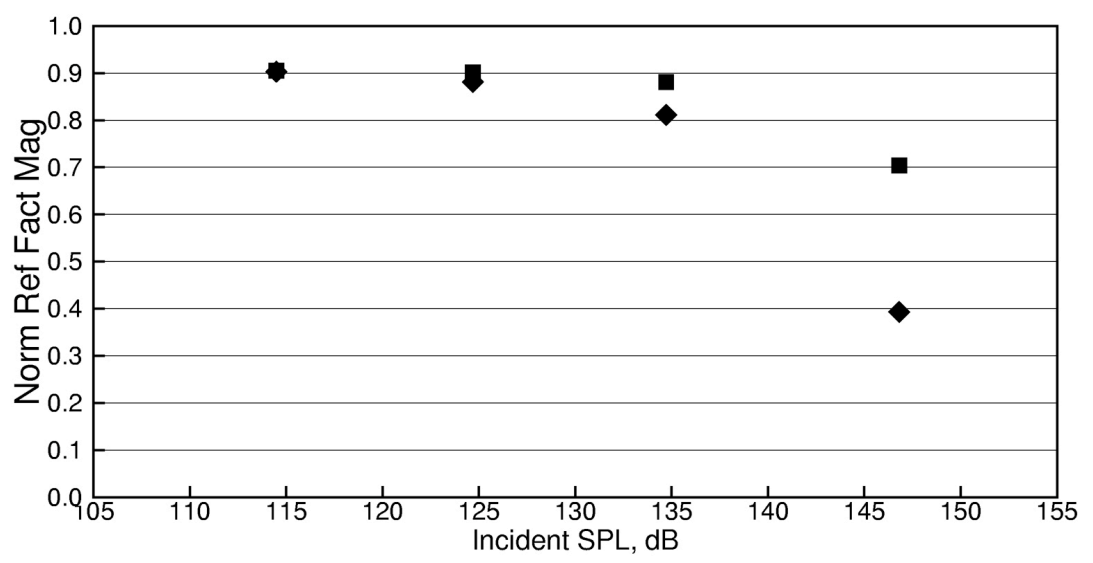

Figure 19. Normalized reflection factor magnitude at different sound pressure levels with 32-slit sample; incident sound frequency of $2.5 \mathrm{kHz}$. $\diamond$, experiment; $\mathbf{\square}$, simulation. 


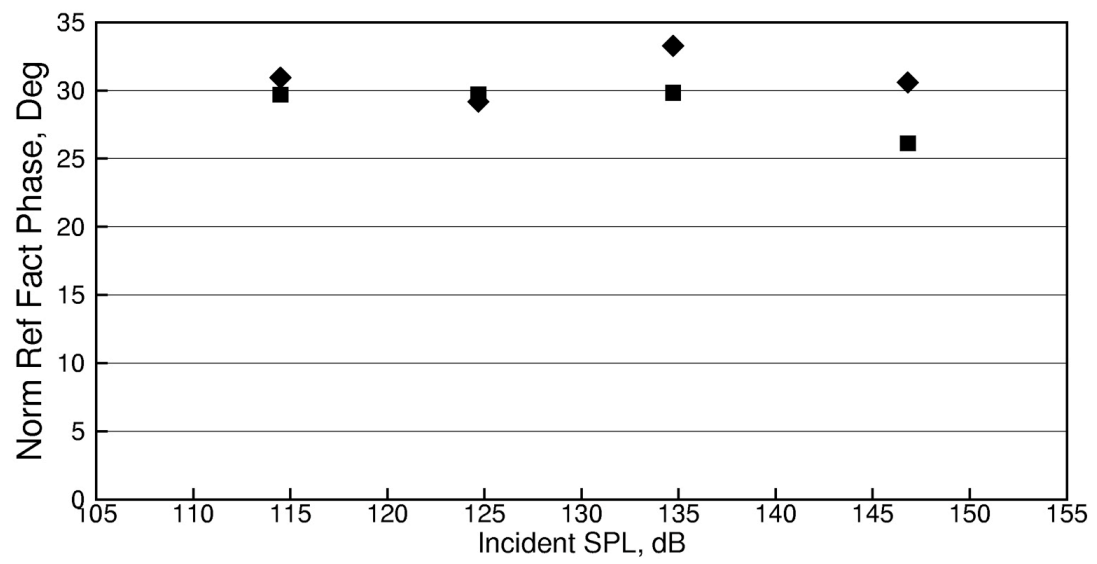

Figure 20. Normalized reflection factor phase at different sound pressure levels with 32-slit sample; incident sound frequency of $2.5 \mathrm{kHz}$. $\diamond$, experiment; $\mathbf{\square}$, simulation.

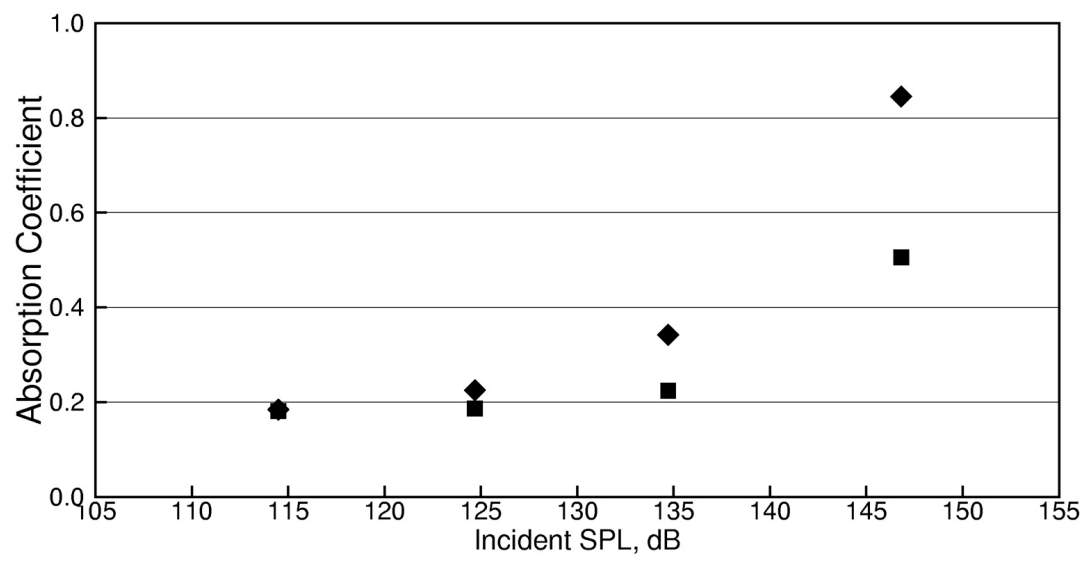

Figure 21. Absorption coefficient at different sound pressure levels with 32-slit sample; incident sound frequency of $2.5 \mathrm{kHz}$. $\diamond$, experiment; $\mathbf{\square}$, simulation.

A similar series of validation tests was performed at a sound frequency of $2500 \mathrm{~Hz}$. Figs. $17-21$ provide the measured and computed liner impedance (resistance and reactance), reflection factor (magnitude and phase), and absorption coefficient as a function of incident sound pressure level. The comparisons of measured and computed impedance (resistance and reactance) are improved for this frequency at the lower incident SPLs, but degrade at the higher SPLs. The reflection factor (magnitude and phase) and absorption coefficient comparisons follow similar trends, again indicating at least a portion of the disparity between measured and computed results are due to a phase mismatch. 


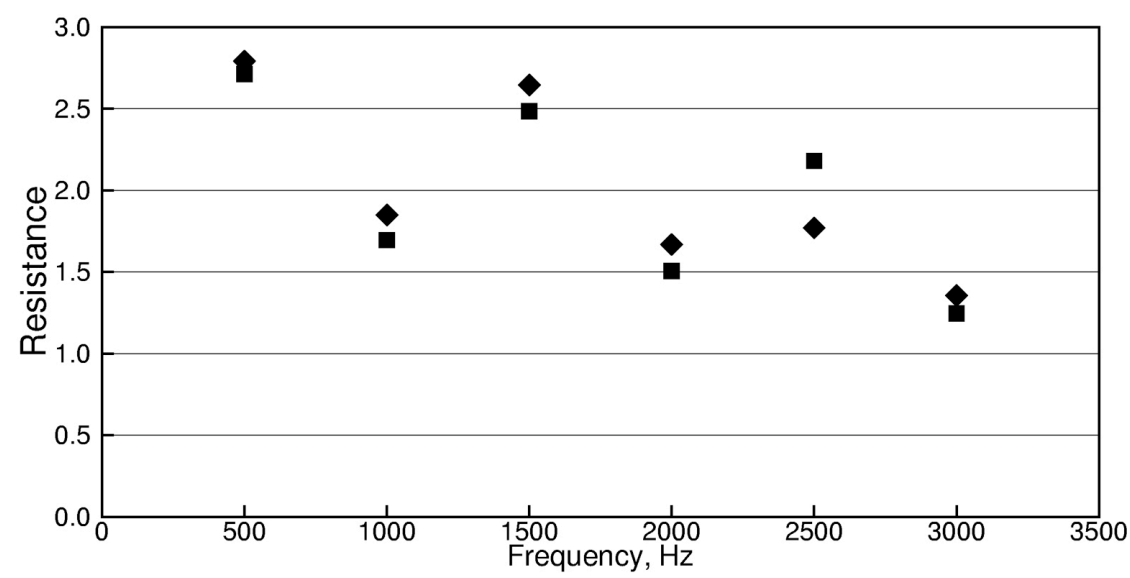

Figure 22. Normalized acoustic resistance at different frequencies with 32-slit sample; incident sound pressure level of $146 \mathrm{~dB}$. $\diamond$, experiment; $\mathbf{\square}$, simulation.

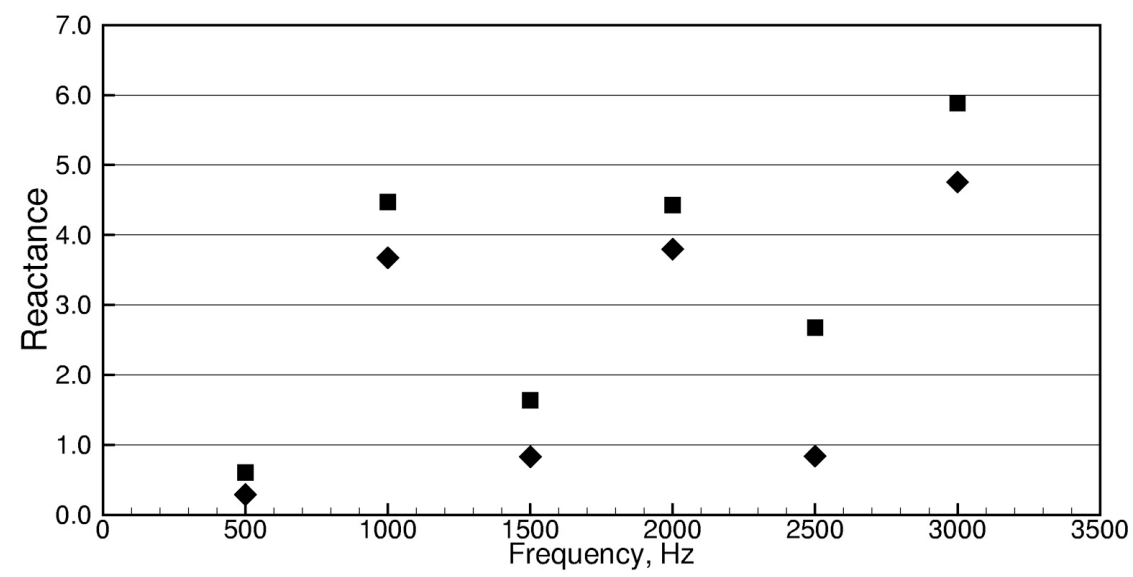

Figure 23. Normalized acoustic reactance at different frequencies with 32-slit sample; incident sound pressure level of $146 \mathrm{~dB}$. $\diamond$, experiment; $\mathbf{\square}$, simulation.

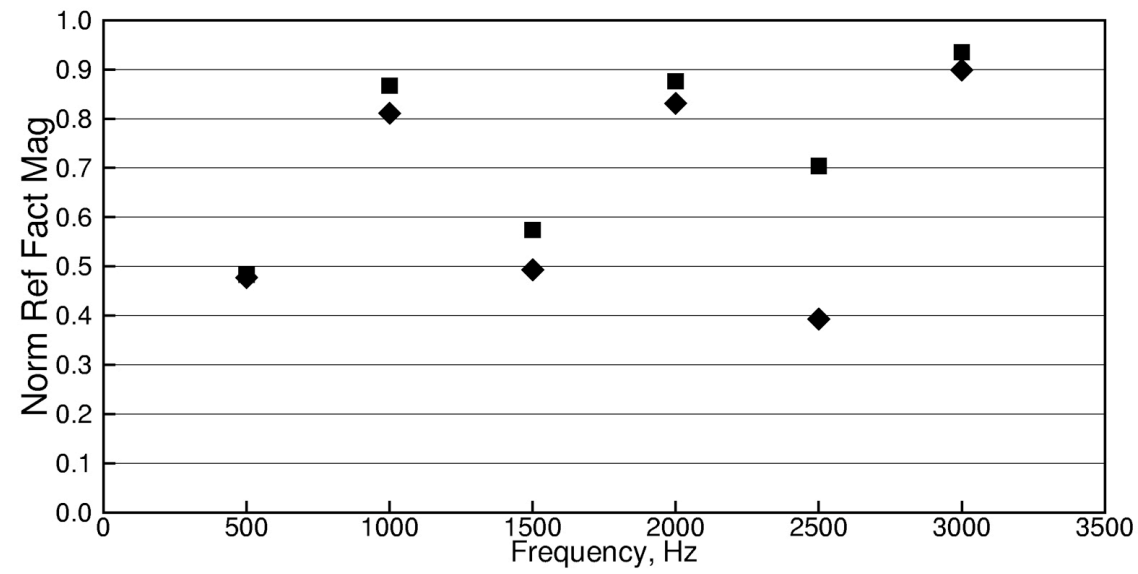

Figure 24. Normalized reflection factor magnitude at different frequencies with 32-slit sample; incident sound pressure level of $146 \mathrm{~dB}$. $\diamond$, experiment; $\mathbf{\square}$, simulation. 


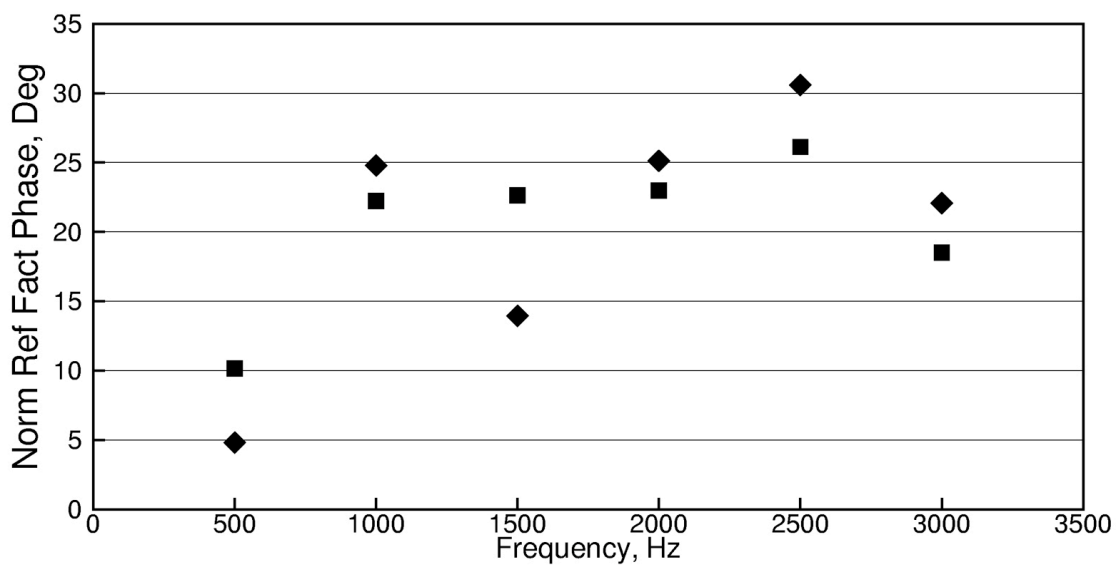

Figure 25. Normalized reflection factor phase at different frequencies with 32-slit sample; incident sound pressure level of $146 \mathrm{~dB}$. $\diamond$, experiment; $\mathbf{\square}$, simulation.

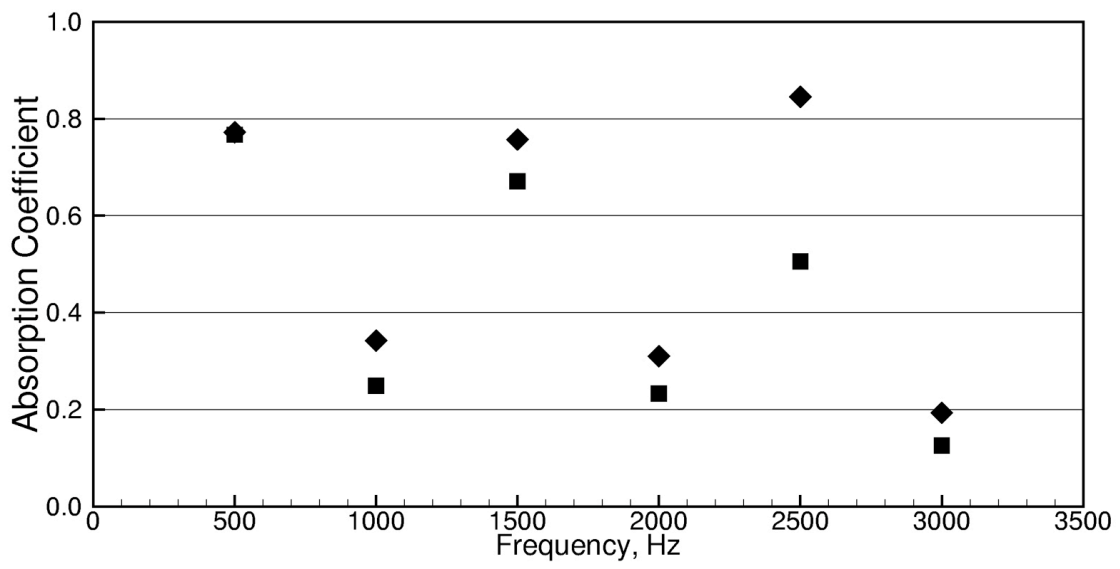

Figure 26. Normalized reflection factor phase at different frequencies with 32-slit sample; incident sound pressure level of $146 \mathrm{~dB}$. $\diamond$, experiment; $\mathbf{\square}$, simulation.

Finally, data were acquired with Sample 6 for a variety of frequencies, with the incident SPL held constant at $146 \mathrm{~dB}$. It is evident from Fig. 23 (reactance) that the cavity resonance is the dominant factor $(-\cot (\mathrm{kL})$ behavior). In general, the comparison of measured and computed resistance is quite acceptable, except at $2500 \mathrm{~Hz}$. However, the differences between measured and computed reactances are greater than 0.5 for most of the test frequencies. Again, a review of the reflection factor magnitude and phase results (Figs. 24 and 25) provides insight. The magnitudes are matched to within 0.1 for all but $2500 \mathrm{~Hz}$, yet the phase differences hover at or above 5 degrees (interestingly, smaller at $2500 \mathrm{~Hz}$ ). Clearly, this phase mismatch must be addressed in further investigations.

\section{Sample 5 (16 slits) results}

Two test series were performed for the 16-slit sample. First, the frequency was held constant at $2 \mathrm{kHz}$ and the incident SPL was varied. Next, the incident SPL was held constant at $150 \mathrm{~dB}$ and the frequency was varied from 500 to $3000 \mathrm{~Hz}$. In general, the comparisons achieved with this sample were very similar to those achieved with the 32-slit sample. 


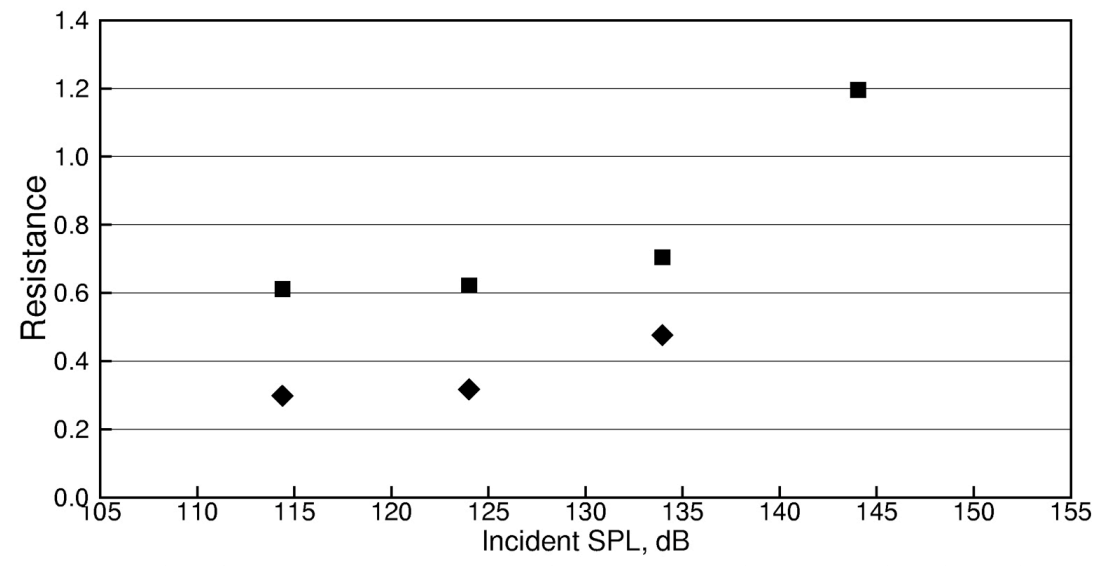

Figure 27. Normalized acoustic resistance at different sound pressure levels with 16-slit sample; incident sound frequency of $2 \mathrm{kHz}$. $\diamond$, experiment; $\mathbf{\square}$, simulation.

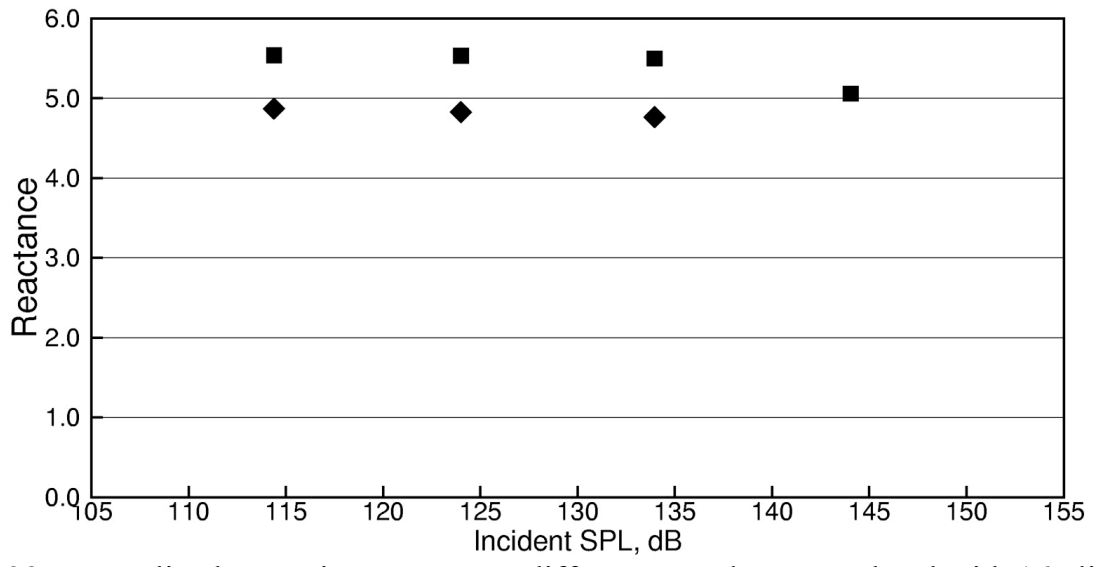

Figure 28. Normalized acoustic reactance at different sound pressure level with 16-slit sample; incident sound frequency of $2 \mathrm{kHz}$. $\diamond$, experiment; $\mathbf{\square}$, simulation.

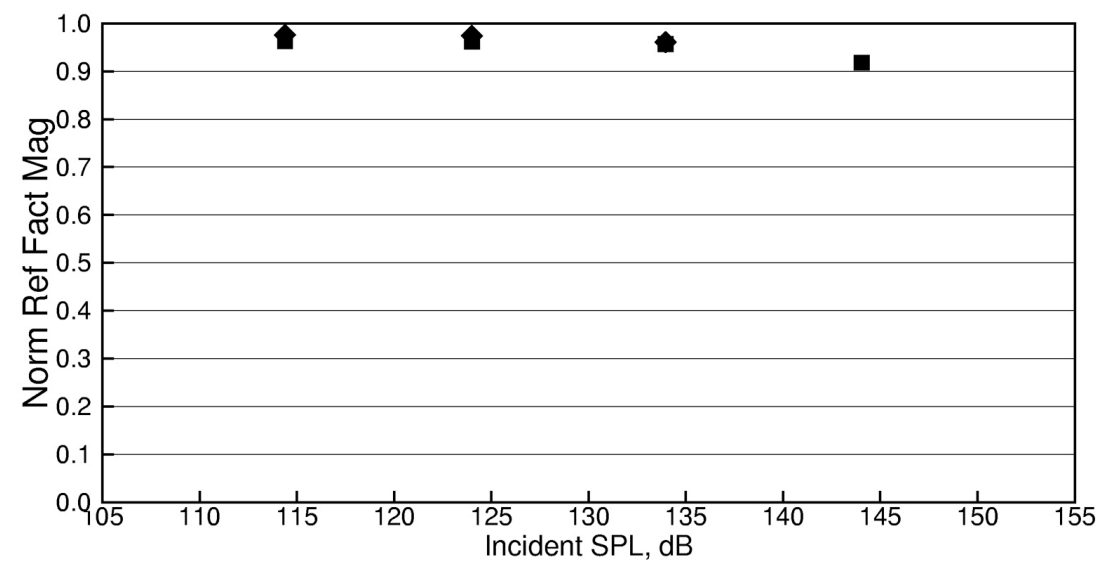

Figure 29. Normalized reflection factor magnitude at different sound pressure levels with 16-slit sample; incident sound frequency of $2 \mathrm{kHz}$. $\diamond$, experiment; $\mathbf{\square}$, simulation. 


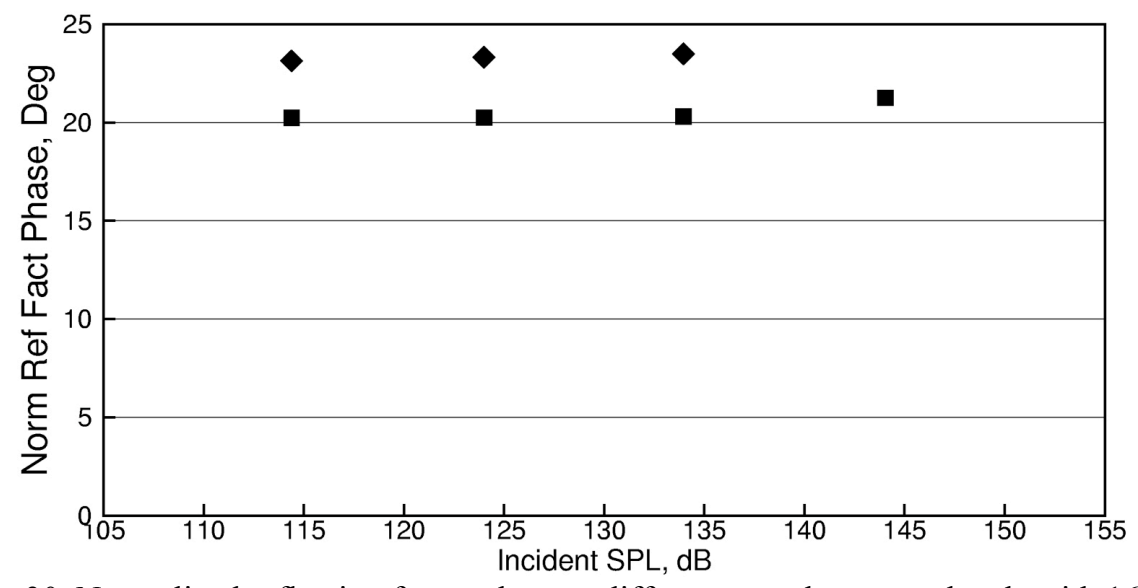

Figure 30. Normalized reflection factor phase at different sound pressure levels with 16-slit sample; incident sound frequency of $2 \mathrm{kHz}$. $\diamond$, experiment; $\mathbf{\square}$, simulation.

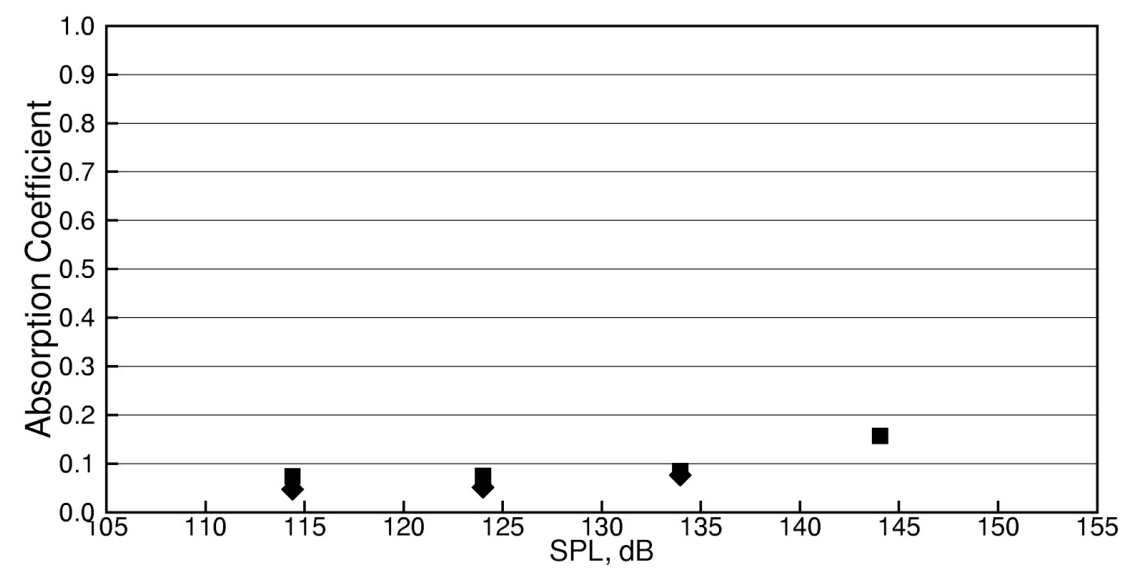

Figure 31. Absorption coefficient at different sound pressure levels with 16-slit sample; incident sound frequency of $2 \mathrm{kHz}$. $\diamond$, experiment; $\mathbf{\square}$, simulation.

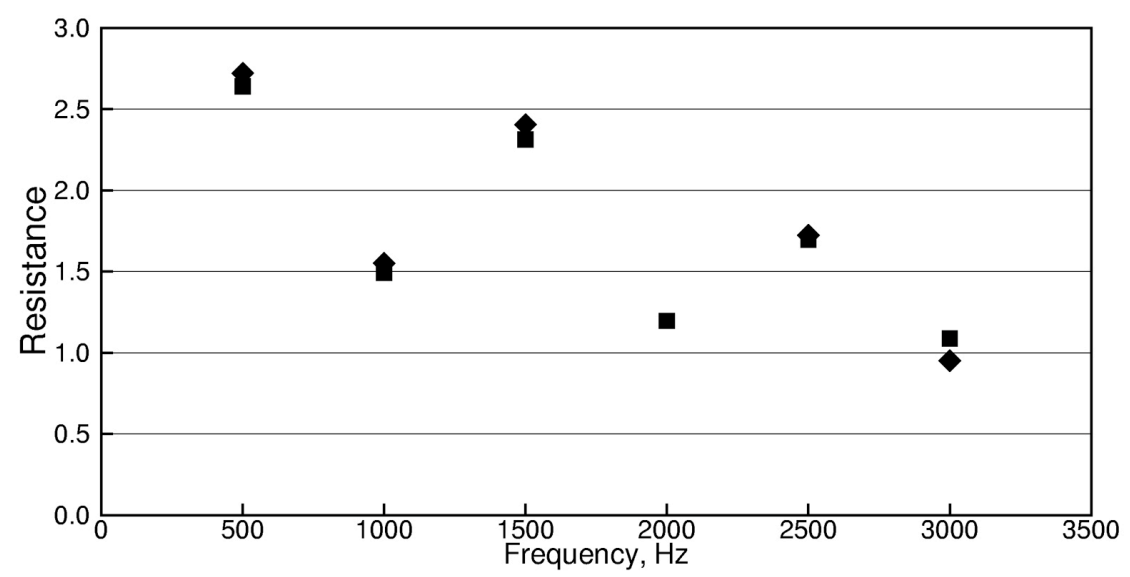

Figure 32. Normalized acoustic resistance at different frequencies with 16-slit sample; incident sound pressure level of $150 \mathrm{~dB}$. $\diamond$, experiment; $\mathbf{\square}$, simulation. 


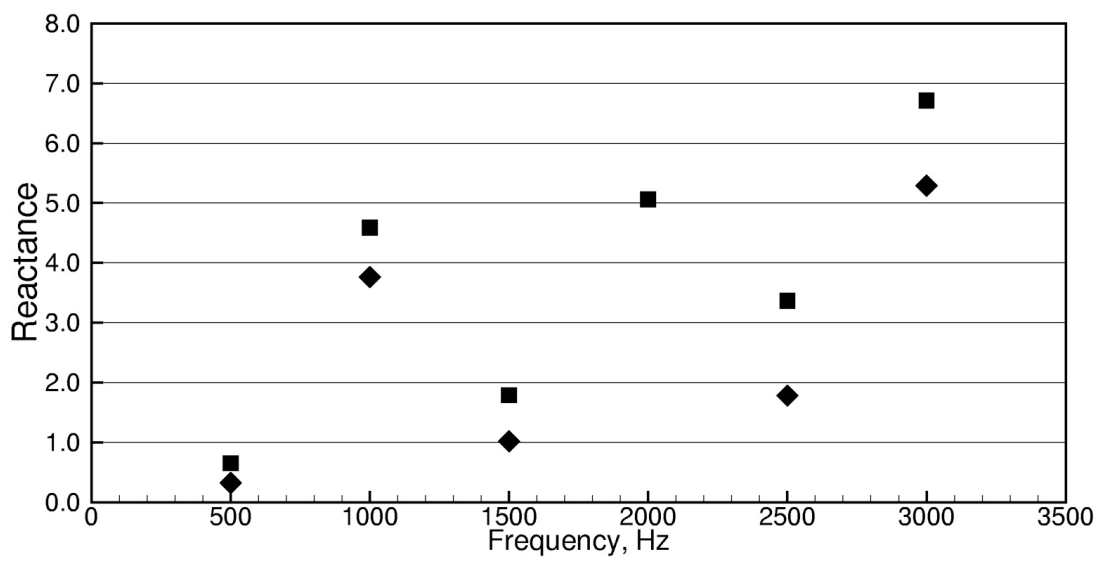

Figure 33. Normalized acoustic reactance at different frequencies with 16-slit sample; incident sound pressure level of $150 \mathrm{~dB}$. $\diamond$, experiment; $\boldsymbol{\square}$, simulation.

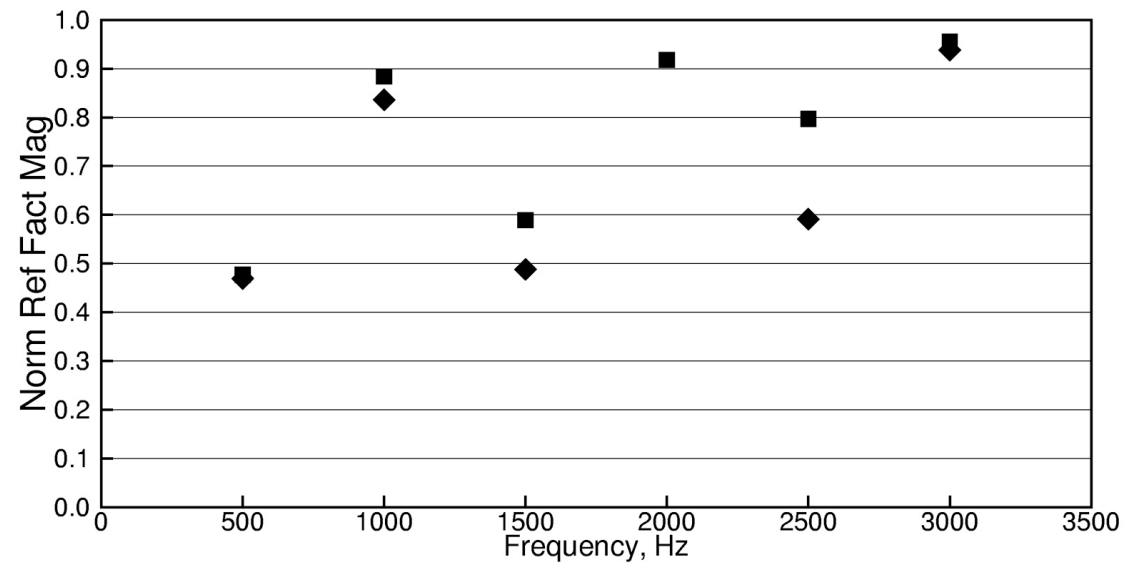

Figure 34. Normalized reflection factor magnitude at different frequencies with 16-slit sample; incident sound pressure level of $150 \mathrm{~dB}$. $\diamond$, experiment; $\mathbf{\square}$, simulation.

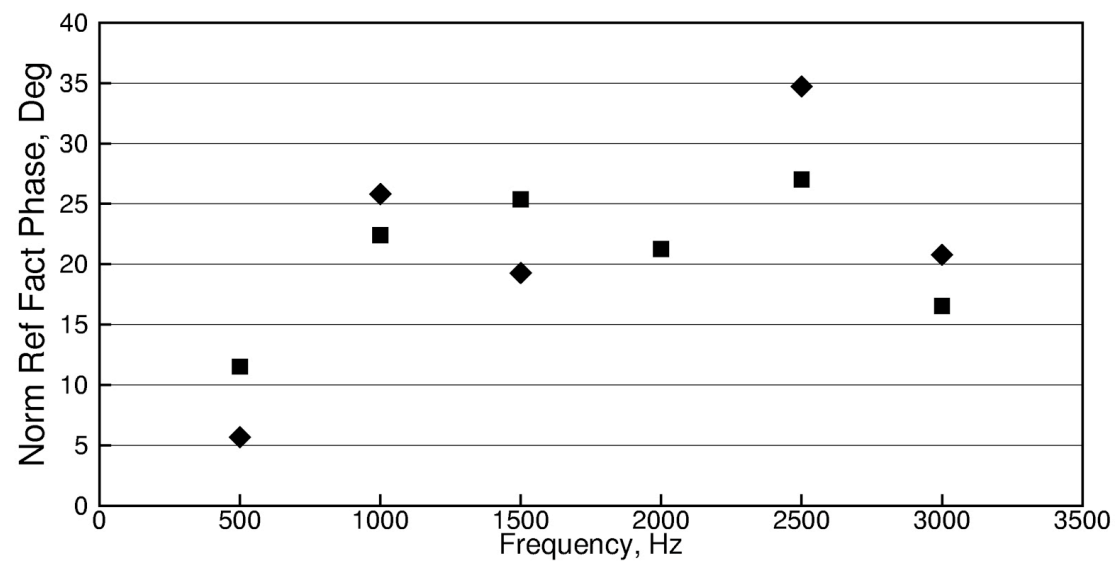

Figure 35. Normalized reflection factor phase at different frequencies with 16-slit sample; incident sound pressure level of $150 \mathrm{~dB}$. $\diamond$, experiment; $\mathbf{\square}$, simulation. 


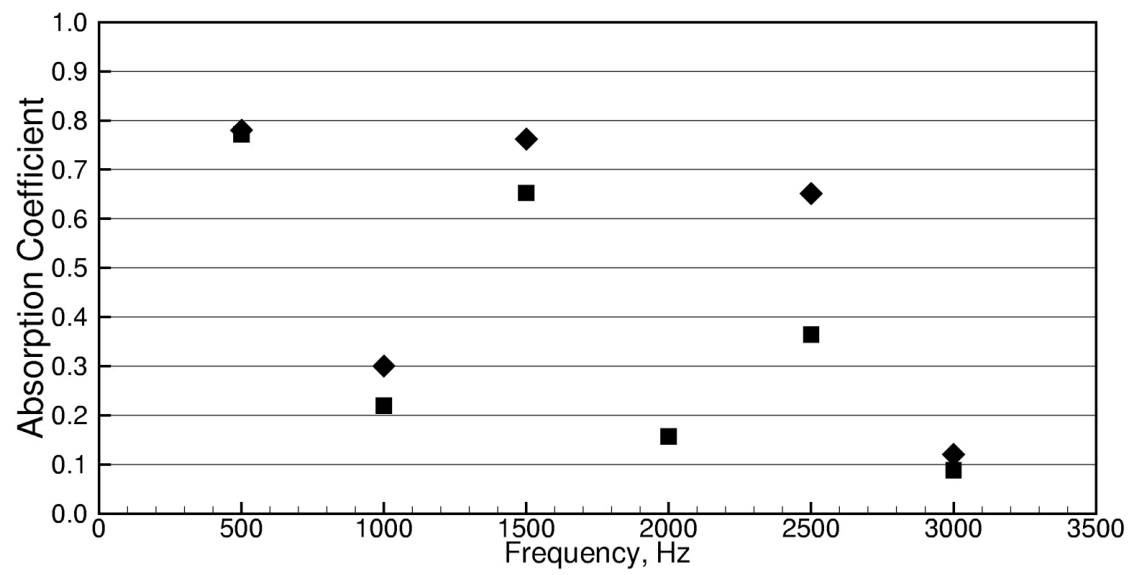

Figure 36. Absorption coefficient at different frequencies with 16-slit sample; incident sound pressure level of $150 \mathrm{~dB}$. $\diamond$, experiment; $\mathbf{\square}$, simulation.

\section{B. Broadband Incident Acoustic Waves}

Two normal incidence impedance tube experiments were conducted using broadband incident sound waves. In these experiments, Sample 6 was used as the face sheet of the resonator. Because of reflection from the face sheet of the resonator, it is very difficult to produce incident acoustic waves with a prescribed spectrum experimentally. For the two experiments reported below, the acoustic drivers were used to generate a broadband spectrum. The acoustic field inside the impedance tube was measured by the two-microphone method. The incident sound wave spectrum was then deduced by analyzing the output of the two microphones. This spectrum was then reproduced by the energy conserving discretization method, discussed in an earlier section, for use as input in direct numerical simulation.

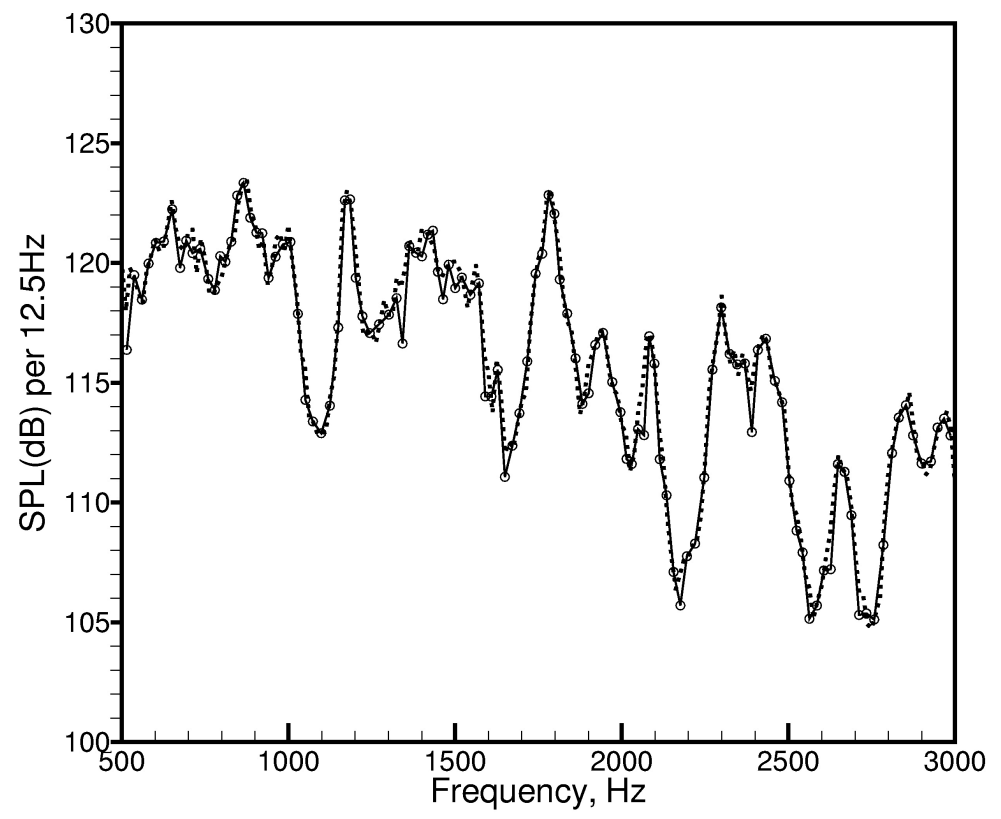

Figure 37. Incident broadband noise spectrum at $141 \mathrm{~dB}$ OASPL from the NASA experiment.

Fig. 37 shows the measured incident broadband noise spectrum at 141 OASPL (dotted line). The spectrum covers the range of $500 \mathrm{~Hz}$ to $3000 \mathrm{~Hz}$. In the numerical simulation, the spectrum was divided into 118 bands. The circles in Fig. 37 are the center frequencies of the bands. As can be seen the discretized spectrum (full line) is a very close approximation of that of the experiment. 


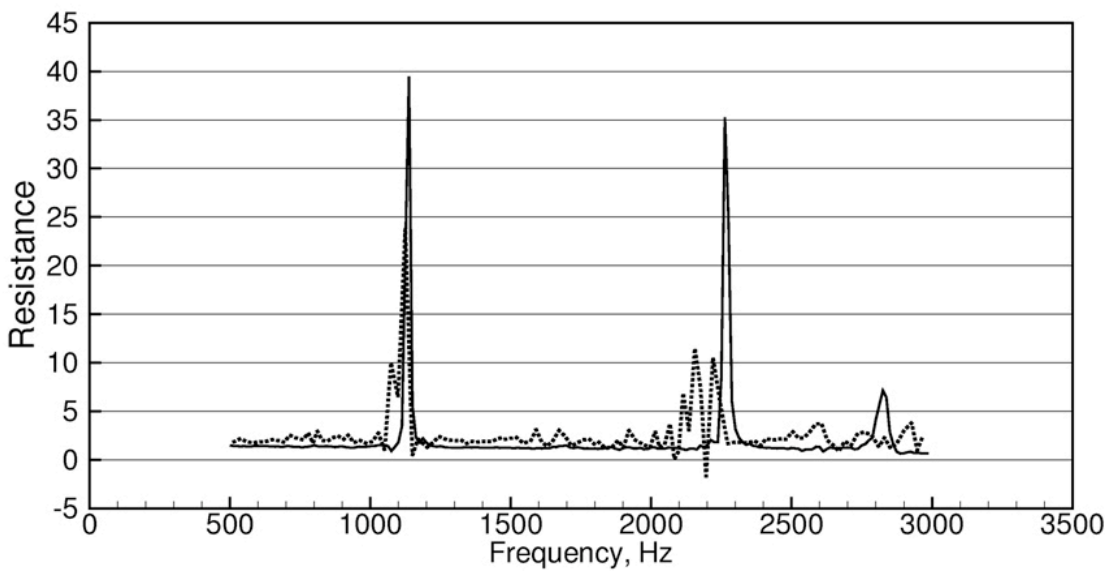

Figure 38. Comparison of measured and computed resistance spectra. OASPL $=141 \mathrm{~dB}$.

Full line - experiment. Dotted line - numerical simulation.

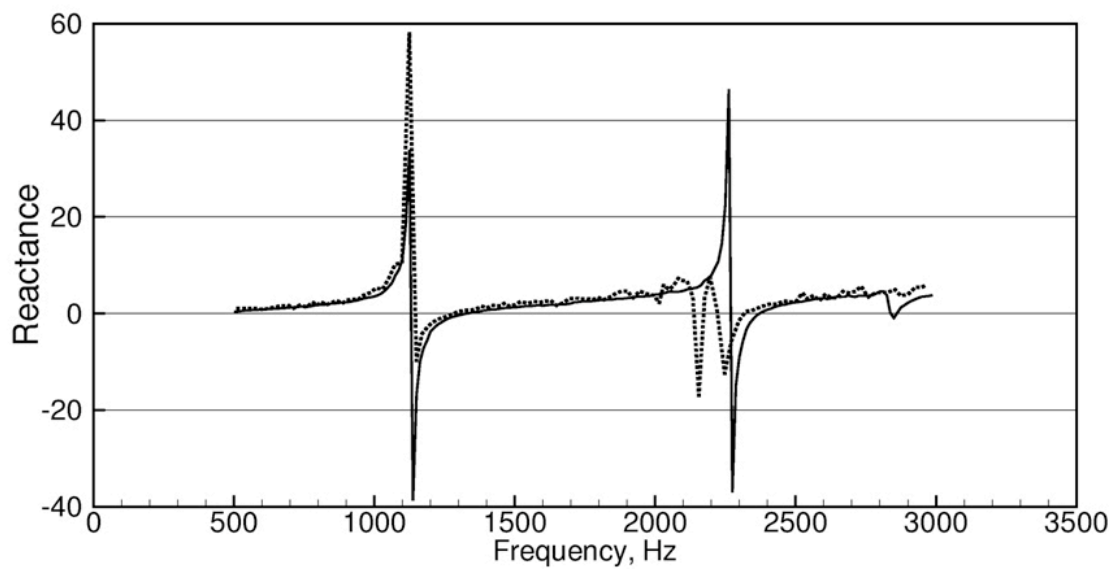

Figure 39. Comparison of measured and computed reactance spectra. OASPL $=141 \mathrm{~dB}$. Full line - experiment. Dotted line - numerical simulation.

Figs. 38 and 39 show the measured resistance and reactance (full line) in the frequency range of $500 \mathrm{~Hz}$ to $3000 \mathrm{~Hz}$. The computed resistance and reactance spectra (dotted line) are also shown. As can be seen, the computed and experimental results follow very similar trends. The numerical simulation reasonably captures the resistance spike that occurs at the first anti-resonance. Similarly, the large drop in reactance that occurs at this frequency is also captured (see Fig. 39). However, the second anti-resonance near $2200 \mathrm{~Hz}$ is not properly captured. The authors have not yet found the source of this disparity. However, the agreement between measured and computed impedances is improved away from these anti-resonance frequencies. It should be noted that they differ by at least 1.0 at some frequencies. Regardless, the overall comparison is quite good, especially given the complexity of the computational process. 


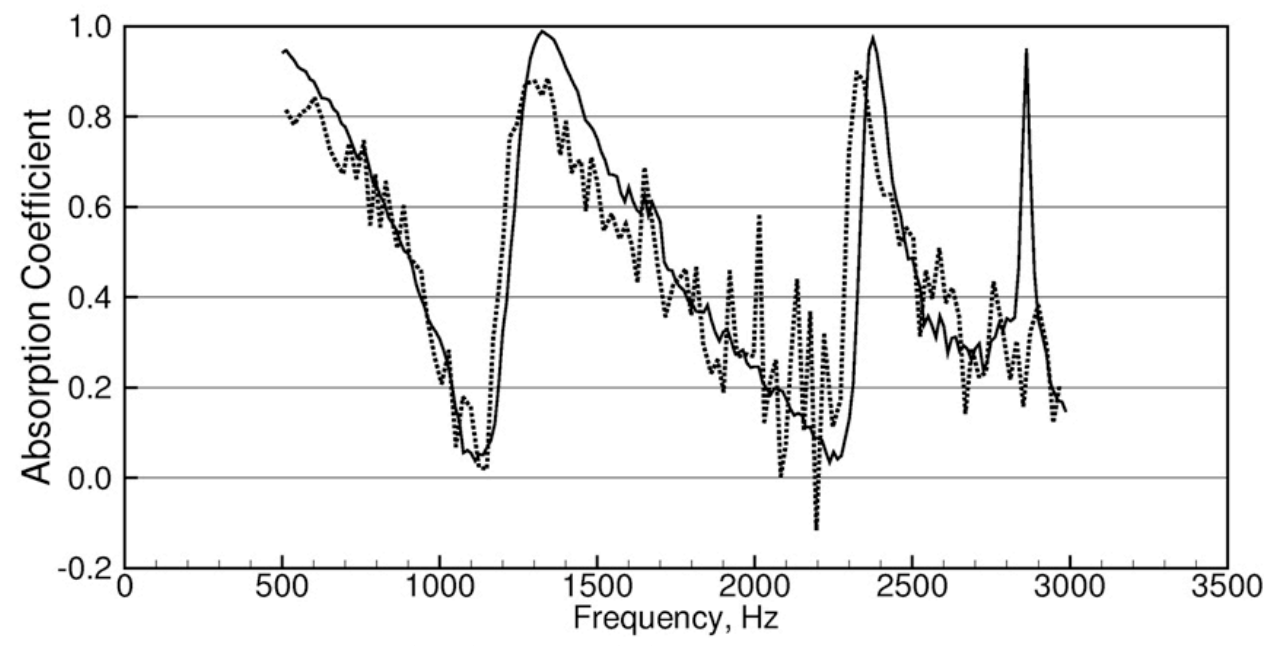

Figure 40. Comparison of measured and computed absorption coefficient spectra. OASPL $=141 \mathrm{~dB}$.

Full line - experiment. Dotted line - numerical simulation.

Fig. 40 shows a comparison between the measured and computed absorption coefficient spectra. There is reasonable agreement up to $2700 \mathrm{~Hz}$. The measured data is relatively smooth. In contrast, the computed curve is jagged. It is believed that the sample length of the computed data used to compute the absorption coefficient is only marginally sufficient. Had a longer sample length been simulated, the result would be a much smoother curve. For future investigations, it is also suggested that multiple simulations with extended length be conducted, such that the individual spectra are smoother and the uncertainty associated with the simulation of broadband data can be evaluated statistically.

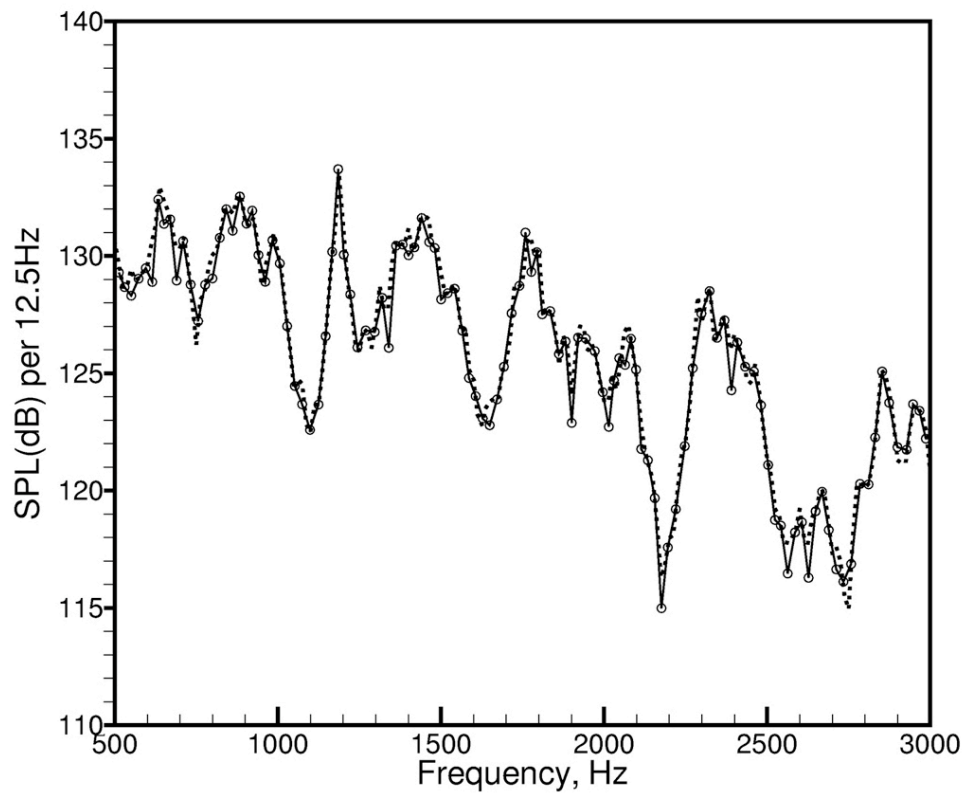

Figure 41. Incident broadband noise spectrum at 150.4 dB OASPL from the NASA experiment.

Dotted line - measured, Circles - numerical simulation.

The same experiment and numerical simulation was repeated at a higher incident sound pressure level. Fig. 41 shows the measured incident sound spectrum at an OASPL of $150.4 \mathrm{~dB}$ (dotted line). The spectrum is divided into 117 bands for numerical simulation. The band center frequencies are indicated by circles in this figure. Figures 42, 43 and 44 show comparisons of the resonator resistance, reactance and absorption coefficient spectra between experimental measurement and numerical simulation results. The agreements are similar to those at $141 \mathrm{~dB}$ OASPL. In this case, the numerical results of reactance, Fig. 43, have two anti-resonances at about the same frequencies as 
the experiment. The peak levels are, however, a bit low. Other than the anti-resonances, there is fairly good agreement over the entire frequency range considered.

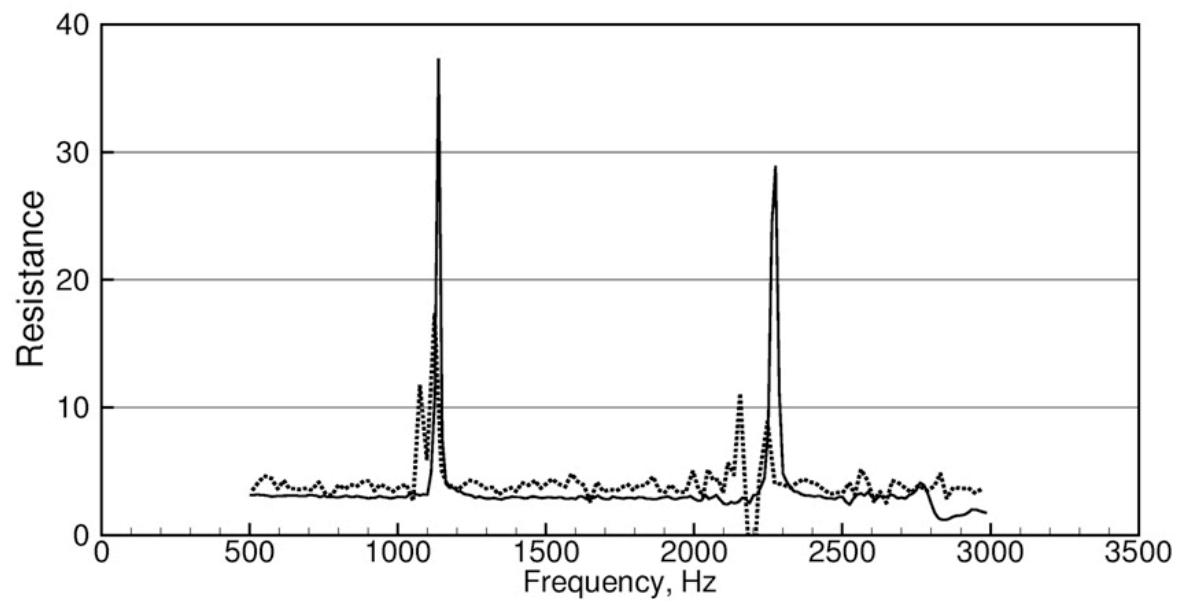

Figure 42. Comparison of measured and computed resistance spectra. OASPL $=150.4 \mathrm{~dB}$. Full line - experiment. Dotted line - numerical simulation.

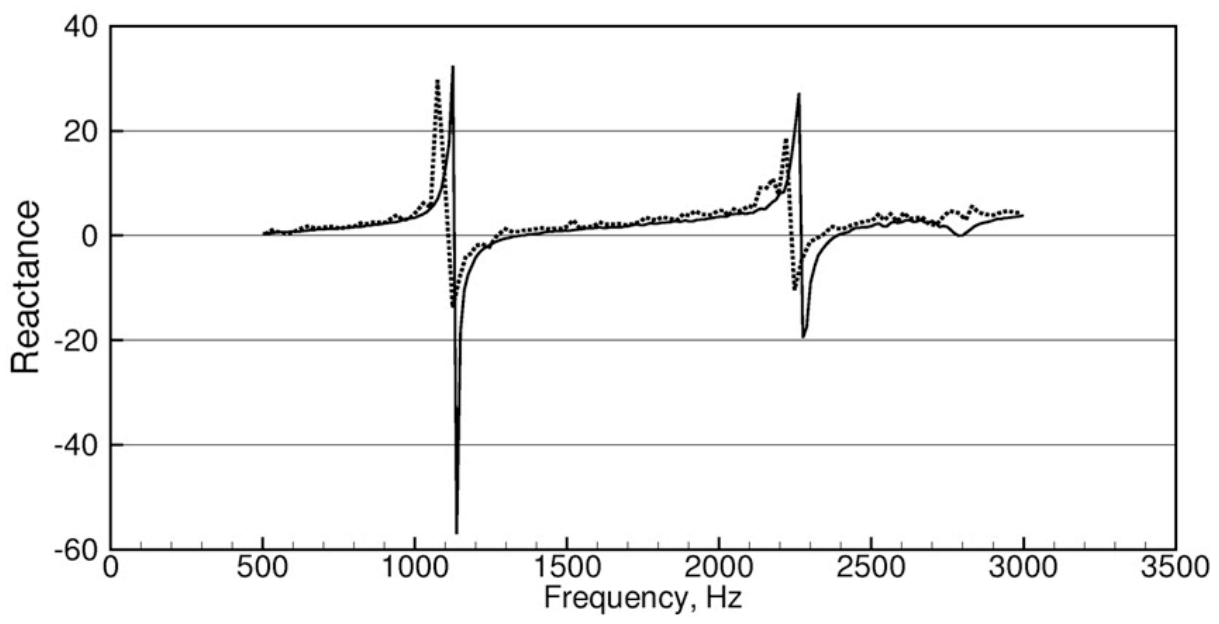

Figure 43. Comparison of measured and computed reactance spectra. OASPL $=150.4 \mathrm{~dB}$. Full line - experiment. Dotted line - numerical simulation.

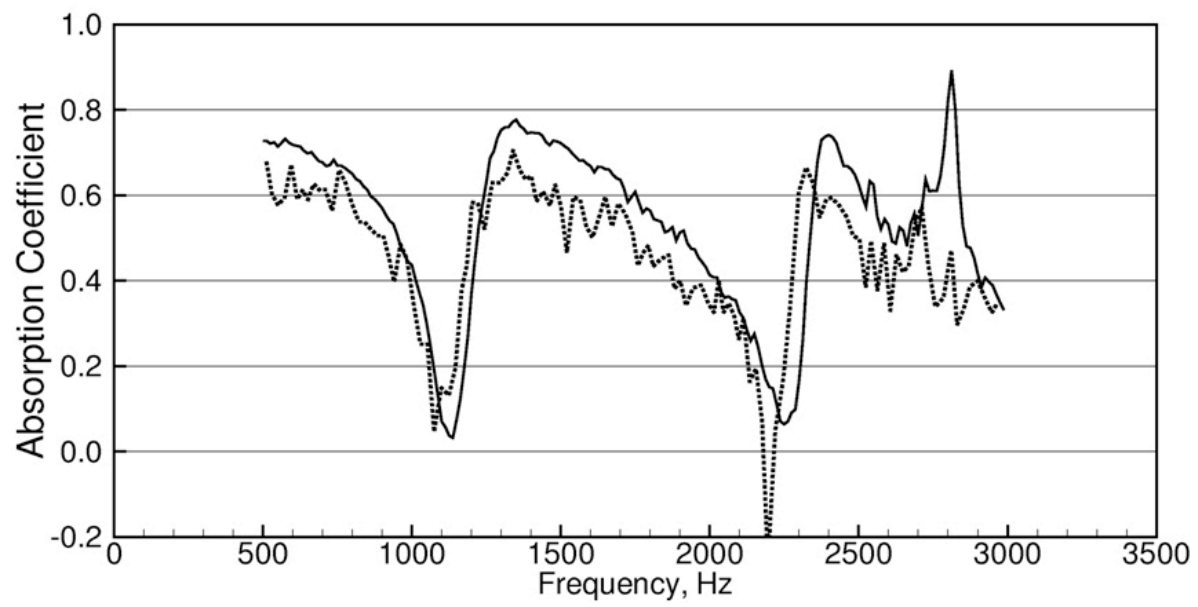

Figure 44. Comparison of measured and computed absorption coefficient spectra. OASPL $=150.4 \mathrm{~dB}$. Full line - experiment. Dotted line - numerical simulation. 


\section{Effect of Slit Aspect Ratio}

By keeping the porosity ratio and slit width fixed, it is clear that an increase in the number of slits would lead to a decrease in the aspect ratio. On the other hand, the total length of the perimeter of the slit openings would increase. This, invariably, would have an impact on the acoustic performance of a resonant liner. In this study, computed results are available at slit aspect ratio of 40 (Sample 1), 2.5 (Sample 5) and 1.25 (Sample 6). Figs. 45 and 46 show the change in resistance and reactance over the SPL range of $114 \mathrm{~dB}$ to $145 \mathrm{~dB}$ due to a change in slit aspect ratio at a frequency of $2000 \mathrm{~Hz}$. It is clear that a decrease in the aspect ratio (i.e., distributing the open area among an increased number of slits) results in an increase in resistance and a decrease in reactance over the entire SPL range included in this investigation. Fig. 47 shows the corresponding increase in absorption coefficient. At the low SPL range of the study (around $114 \mathrm{~dB}$ ), there is no vortex shedding, and the damping of incident acoustics waves is largely due to wall friction. As the open area is subdivided into a larger number of small slits, the total perimeter of all the slits is larger than that of the individual large slit. Thus, one would expect larger dissipation and hence an increase in absorption coefficient with longer perimeter of slit openings.

At high SPL, the dominant dissipation mechanism is vortex shedding. This causes the resistance of the single slit (aspect ratio of 40) to increase at this SPL. As the slits become smaller, the sample thickness-to-slit width ratio increases. Under this condition, the viscous dissipation due to the wall friction increases. When combined with the dominant vortex shedding at high SPL, this results in further resistance increases, which leads to increased absorption coefficient.

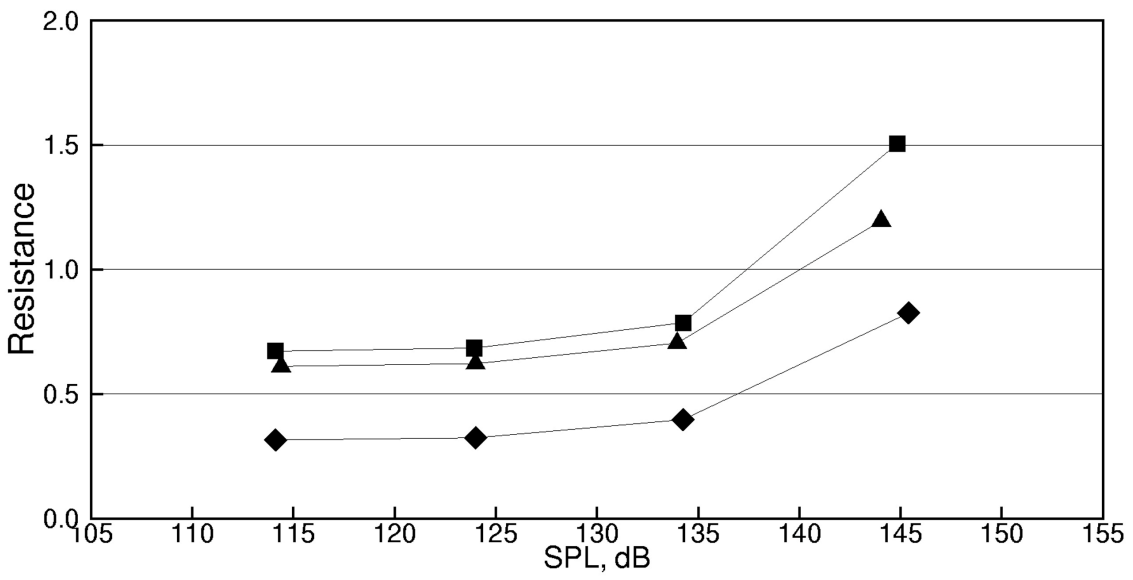

Figure 45. Variation of liner resistance with slit aspect ratio at $2000 \mathrm{~Hz}$ frequency. $\bullet, \boldsymbol{\Lambda}, \boldsymbol{\square}$ slit aspect ratio, 40, 2.5, 1.25.

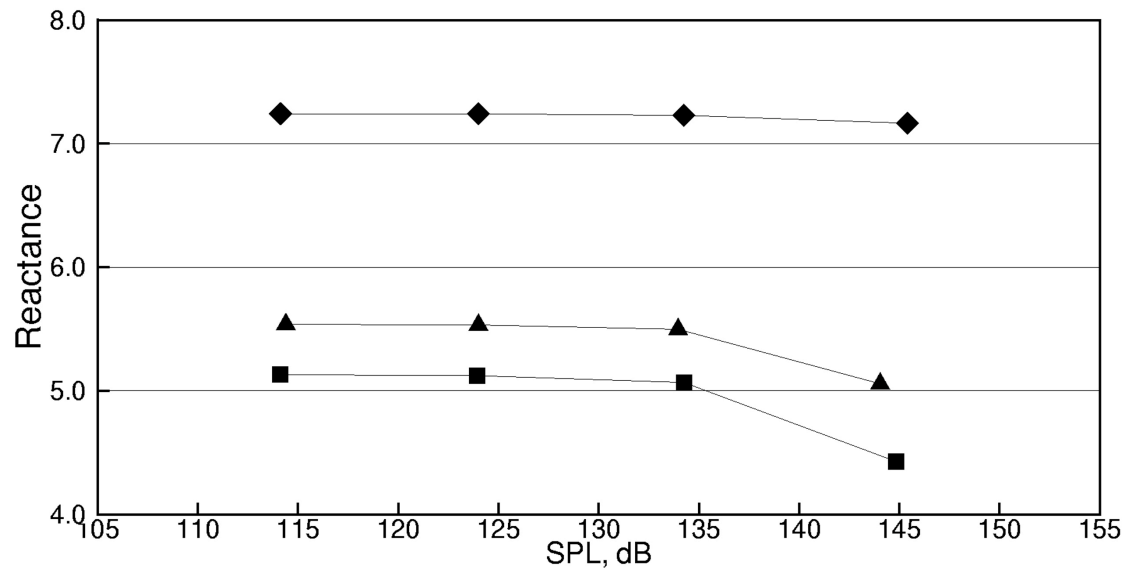

Figure 46. Variation of liner reactance with slit aspect ratio at $2000 \mathrm{~Hz}$ frequency. $\bullet, \boldsymbol{\Delta}, \mathbf{\square}$ slit aspect ratio, 40, 2.5, 1.25. 


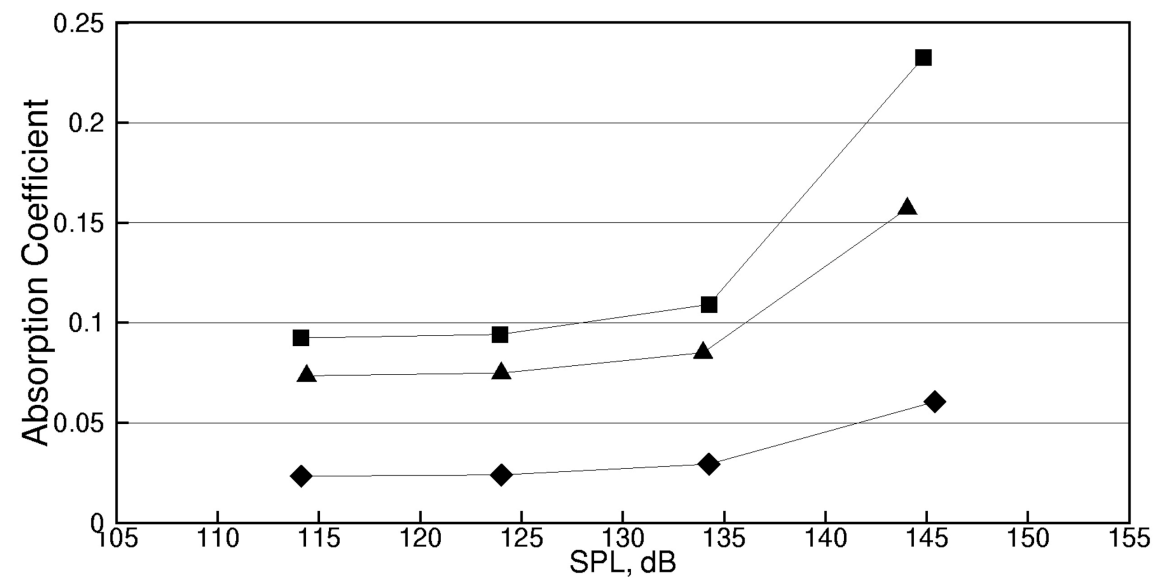

Figure 47. Variation of liner absorption coefficient with slit aspect ratio at $2000 \mathrm{~Hz}$ frequency.

$\diamond, \boldsymbol{\Delta}, \mathbf{\square}$ slit aspect ratio, $40,2.5,1.25$.

\section{Vortex Shedding and Behavior of Shed Vortices}

One advantage of numerical simulation is that it provides a full set of spatial and temporal data. We make use of this data to observe the vortex-shedding phenomenon at the resonator opening. For convenience of observation, a number of videos on the fluid motion in the vicinity of the resonator are made. They show the motion of the vortices in different planes that cut through the normal incidence impedance tube. Vortex motion under the excitation of single frequency incident sound waves and broadband incident sound waves are recorded and analyzed. A short summary of the observed vortex behavior is provided below.

\section{A. Single Frequency Incident Sound Waves}

When a vortex is shed, its plan form initially has the same shape as the resonator opening. It is not circular. However, after the vortex moves away from the resonator opening, it becomes a relatively free vortex. It is observed that such a vortex has the tendency to adjust itself to become circular. The large dimension of the vortex ring gradually reduces. This can be seen in Fig. 48. This figure shows the density distribution in a plane cutting the larger width of the slit (Sample 5). In this figure, the width of the vortex farther away from the resonator opening is smaller than that of the vortex just shed. On the other hand, the small dimension of the vortex ring increases. This is shown in Fig. 49. This figure corresponds to that of a plane cutting through the smaller dimension of the slit. It is easy to observe in this figure that the width of the vortex farther away from the resonator opening is larger than that of the vortex just shed. At this time, it is not clear why a free vortex tends to become circular. The spinning motion apparently contributes to this evolution into a circular form. It also appears that a circular vortex is the natural stable configuration. 


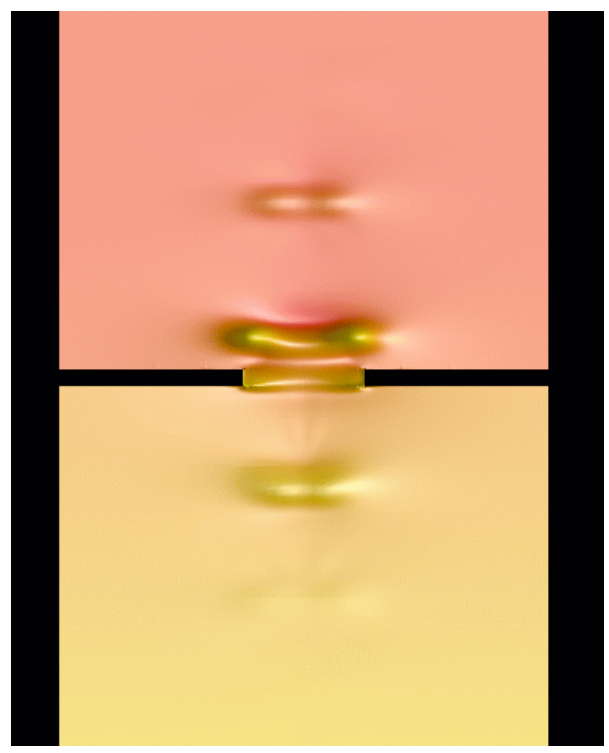

Figure 48. Vortex motion as viewed in a plane cutting through the larger side of the slit opening.

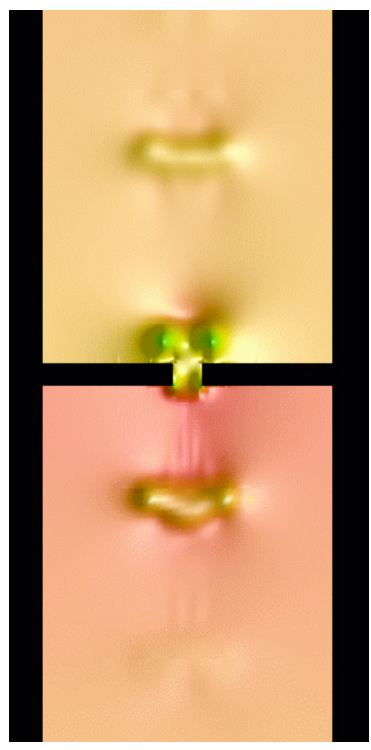

Figure 49. Vortex motion as viewed in a plane cutting through the smaller side of the slit opening.

The shed vortices form two vortex trains, one on each side of the resonator opening. The vortices maintain a fairly regular spacing (see Fig. 50). Vortices are shed alternately on the two sides of the face sheet. These vortex trains move away from the resonator opening in opposite directions. It appears that the vortices move in formation due to mutual interaction. The directions of rotation of the vortices on opposite sides of the resonator opening are opposite. This is believed to be the principal reason why the vortex trains move in directions opposite to each other.

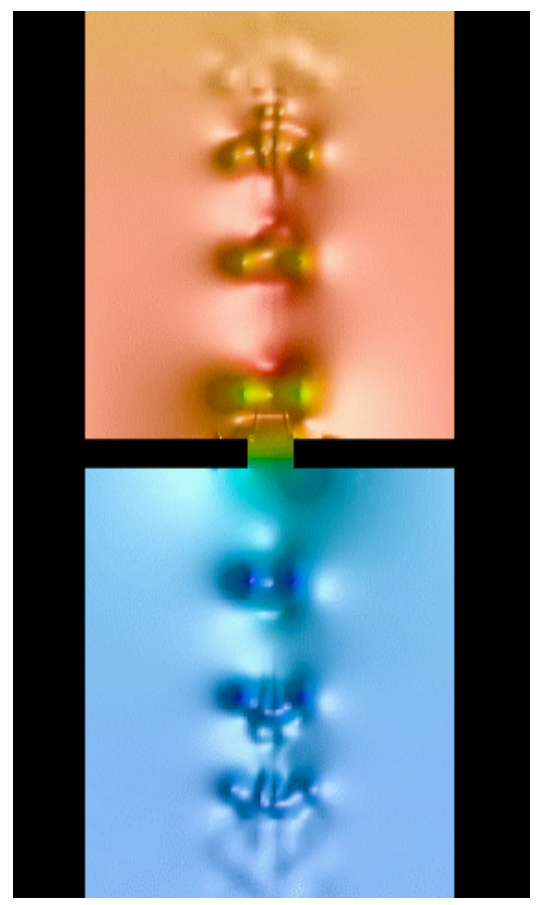

Figure 50. Instantaneous density distribution showing two vortex trains shed from a resonator opening. 


\section{B. Broadband Incident Sound Waves}

When a slit resonator is subjected to excitation by broadband incident sound waves at high SPL, the flow field around the resonator opening is again dominated by vortex shedding. However, vortices are shed randomly (see Fig. 51). In some cases, two vortices are shed on the same side of the resonator opening before a vortex is shed on the other side (see Fig. 52). Vortex shedding is a nonlinear phenomenon. Thus, we should not expect the acoustic characteristics of a liner at a given frequency in the presence of broadband noise to be the same as that of the liner under discrete frequency forcing. In other words, if $Z(\omega)$ is the resonator impedance under broadband incident sound and $Z_{\omega}$ is the impedance of the resonator under a single frequency incident sound wave at the same frequency $\omega$, then, in general $Z(\omega) \neq Z_{\omega}$.

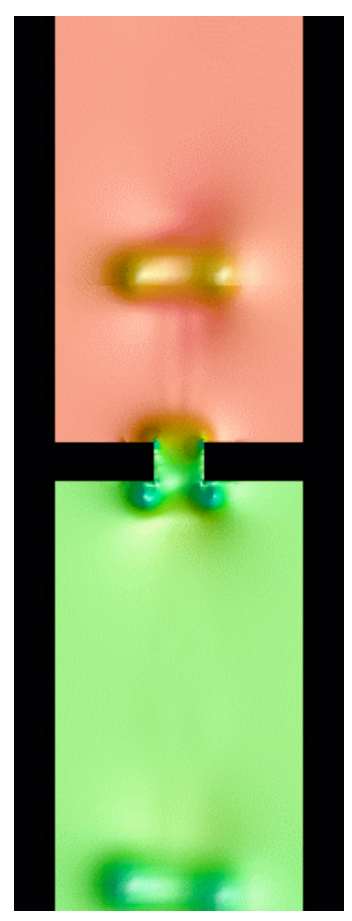

Figure 51. Random vortex shedding under excitation by broadband incident sound waves.

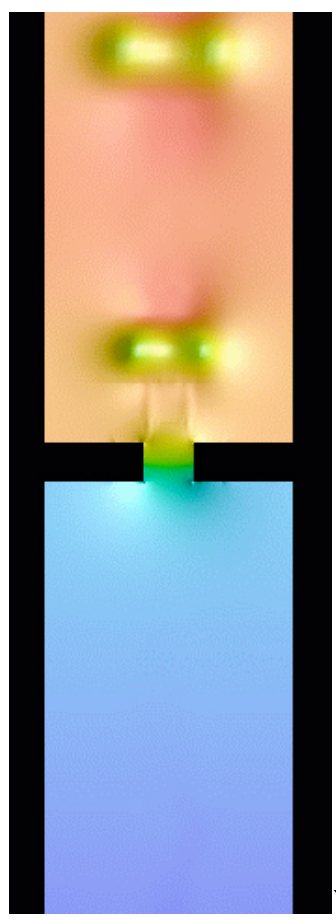

Figure 52. Non-periodic random vortex shedding.

\section{Conclusion and Summary}

One of the principal objectives of the present investigation is to validate, by experimental measurements, three-dimensional direct numerical simulation results of slit resonators in a normal incidence impedance tube. Both discrete and broadband frequency incident sound waves are used. Test cases include slits with substantially different aspect ratios. It has been found the simulation results track the trends of the measured impedances quite well, but do not match to an acceptable tolerance to support the exclusive usage of direct numerical simulation for liner evaluation. However, when these impedance spectra are converted to the corresponding reflection factor magnitude and phase, differences in the computed and measured reflection factor phase appear to be at least partly responsible for the impedance spectra differences. Two potential causes for these phase differences are offered. First, measurements conducted at frequencies near anti-resonance are susceptible to measurement errors, and more detailed measurement processes may be needed. Second, the direct numerical simulation process used herein neglects the effects of the walls of the normal incidence tube, thereby failing to account for higher-order modes that exist in the near field of the resonator openings. Additional computations may be needed in which the entire normal incidence tube is included in the simulation. 
For liners with the same porosity, it is demonstrated that the hole geometry plays a role in determining the impedance of a liner. The absorption coefficient is found to increase with increase in the total perimeter of the resonator openings over the frequency and slit aspect ratio range studied. This is true over a wide range of sound pressure levels. It is known ${ }^{12,16}$ that, at low incident SPL, the principal sound dissipation mechanism is viscous dissipation in the oscillatory boundary layer adjacent to the walls at the resonator openings. Thus, it is not surprising that the increased total perimeter (summed over all of the slits) results in increased dissipation. At high SPL, the principal mechanism is vortex shedding. ${ }^{12,16}$ The acoustic energy dissipation depends on the total rotational kinetic energy imparted to the shed vortices, but the rotational kinetic energy of a vortex depends on both the length and strength of the vortex. This results in an increase in absorption coefficient for the single slit. As the open area is subdivided into multiple slits, each slit has an increased sample thickness-to-slit width ratio, which results in increased viscous dissipation due to the wall friction. When combined with the dominant vortex shedding, this increased viscous dissipation results in an increased absorption coefficient for the larger number of slits (aspect ratio of 1.25).

Finally, the present investigation extends the finding of previous studies to three dimensions that vortex shedding is the dominant sound dissipation mechanism at high sound pressure levels. However, for low aspect ratio slits, the vortex shedding process is highly regular under discrete frequency incident sound excitation. This is unlike the irregular, fairly random vortex shedding for large aspect ratio slits (observed in two-dimensional simulations ${ }^{16}$ ). It is also observed that vortices, once shed and upon becoming relatively free of the influence of nearby walls, tend to readjust themselves into vortices with a circular plan form. The vortex shape readjustment and vortex dynamical interaction are processes that are believed to exert significant influence on the strength of shed vortices and hence liner dissipation rate. The study of vortex dynamics is, however, beyond the scope of the present work.

\section{Acknowledgment}

The work of CKWT and HJ was supported initially by a NASA Cooperative Agreement NNL04AA01A.

\section{Appendix: Energy Conserving Discretization}

In this appendix, we will discuss how to produce the pressure signal of a prescribed noise spectrum in time. Let us first show that the time varying pressure signal given by the mathematical expression below has a spectrum equal to any given spectrum $S(\omega)$ where $\omega$ is the angular frequency:

$$
p(t)=\int_{-\infty}^{\infty}[2 S(\omega)]^{\frac{1}{2}} \cos (\omega t+\chi(\omega)) d \omega
$$

where $\chi(\omega)$ is a random function of $\omega$. The noise spectrum of $p(t)$ given by Eq. (A1) is the Fourier transform of the autocorrelation function. The autocorrelation function is,

$$
\overline{p(t) p(t+\tau)}=\int_{-\infty}^{\infty} \int_{-\infty}^{\infty} 2\left[S\left(\omega^{\prime}\right) S\left(\omega^{\prime \prime}\right)\right]^{\frac{1}{2}} \overline{\cos \left(\omega^{\prime} t+\chi\left(\omega^{\prime}\right)\right) \cos \left(\omega^{\prime \prime} t+\omega^{\prime \prime} \tau+\chi\left(\omega^{\prime \prime}\right)\right)} d \omega^{\prime} d \omega^{\prime \prime} .
$$

The overbar in Eq. (A2) is the time average. Now,

$$
\begin{aligned}
& \overline{\cos \left(\omega^{\prime} t+\chi\left(\omega^{\prime}\right)\right) \cos \left(\omega^{\prime \prime} t+\omega^{\prime \prime} \tau+\chi\left(\omega^{\prime \prime}\right)\right)}=\operatorname{Lim}_{T \rightarrow \infty} \frac{1}{2 T} \int_{-T}^{T} \cos \left(\omega^{\prime} t+\chi\left(\omega^{\prime}\right)\right) \cos \left(\omega^{\prime \prime} t+\omega^{\prime \prime} \tau+\chi\left(\omega^{\prime \prime}\right)\right) d t \\
& =\operatorname{Lim}_{T \rightarrow \infty} \frac{1}{2 T} \int_{-T}^{T}\left\{\cos \left(\omega^{\prime} t+\chi\left(\omega^{\prime}\right)\right)\left[\cos \left(\omega^{\prime \prime} t+\chi\left(\omega^{\prime \prime}\right)\right) \cos \left(\omega^{\prime \prime} \tau\right)-\sin \left(\omega^{\prime \prime} t+\chi\left(\omega^{\prime \prime}\right)\right) \sin \left(\omega^{\prime \prime} \tau\right)\right]\right\} d t
\end{aligned}
$$

Because $\chi(\omega)$ is a random function, in the limit $T \rightarrow \infty$, the right side of Eq. (A3) is zero except when $\omega^{\prime}=\omega^{\prime \prime}$. In this exceptional case, the right side of Eq. (A3) becomes $\frac{1}{2} \cos \left(\omega^{\prime \prime} \tau\right)$. Hence, it is found, 


$$
\overline{\cos \left(\omega^{\prime} t+\chi\left(\omega^{\prime}\right)\right) \cos \left(\omega^{\prime \prime} t+\omega^{\prime \prime} \tau+\chi\left(\omega^{\prime \prime}\right)\right)}=\frac{1}{2} \cos \left(\omega^{\prime \prime} \tau\right) \delta\left(\omega^{\prime}-\omega^{\prime \prime}\right)
$$

where $\delta(\omega)$ is the delta function. Substitution of Eq. (A4) into the right side of Eq. (A2) and upon integrating over $\omega^{\prime \prime}$, it is straightforward to find,

$$
\overline{p(t) p(t+\tau)}=\int_{-\infty}^{\infty} S\left(\omega^{\prime}\right) \cos \left(\omega^{\prime} \tau\right) d \omega^{\prime}
$$

Now, by taking the Fourier transform of autocorrelation function (A5), it is easy to find that the spectrum function is $S(\omega)$. [Note: the customary assumption of $S(-\omega)=S(\omega)$ is adopted].

For computation purpose, we need to discretize the integral on the right side of Eq.(A1) to produce random pressure fluctuation $p(t)$ in real time. To this end, let us divide the spectrum into a large number of narrow bands. Let $\omega_{j}$ and $\Delta \omega_{j}$ be the center frequency and bandwidth of the $\mathrm{j}^{\text {th }}$ band as shown in Fig. 9.

The pressure fluctuation corresponding to the energy in the $\mathrm{j}^{\text {th }}$ band according to Eq. (A1) is,

$$
p_{j}(t)=\int_{\omega_{j}-\frac{1}{2} \Delta \omega_{j}}^{\omega_{j}+\frac{1}{2} \Delta \omega_{j}}[2 S(\omega)]^{\frac{1}{2}} \cos (\omega t+\chi(\omega)) d \omega
$$

The energy, $E_{j}$, in this band is,

$$
E_{j}=\overline{p_{j}^{2}(t)}=\int_{\omega_{j}-\frac{1}{2} \Delta \omega_{j}}^{\omega_{j}+\frac{1}{2} \Delta \omega_{j}} \int\left[2 S\left(\omega^{\prime}\right)\right]^{\frac{1}{2}}\left[2 S\left(\omega^{\prime \prime}\right)\right]^{\frac{1}{2}} \overline{\cos \left(\omega^{\prime} t+\chi\left(\omega^{\prime}\right)\right) \cos \left(\omega^{\prime \prime} t+\chi\left(\omega^{\prime \prime}\right)\right)} d \omega^{\prime} d \omega^{\prime \prime}
$$

In the limit $\Delta \omega_{j} \rightarrow 0$, we may take $S\left(\omega^{\prime}\right)=S\left(\omega^{\prime \prime}\right)=S\left(\omega_{j}\right)$ in the above integral. This gives,

$$
E_{j}=2 S\left(\omega_{j}\right) \int_{\omega_{j}-\frac{1}{2} \Delta \omega_{j}}^{\omega_{j}+\frac{1}{2} \Delta \omega_{j}} \int \overline{\cos \left(\omega^{\prime} t+\chi\left(\omega^{\prime}\right)\right) \cos \left(\omega^{\prime \prime} t+\chi\left(\omega^{\prime \prime}\right)\right)} d \omega^{\prime} d \omega^{\prime \prime}
$$

Upon replacing the integrand by Eq. (A4), the double integral can be evaluated to yield,

$$
E_{j}=S\left(\omega_{j}\right) \Delta \omega_{j}
$$

Now, we will approximate the pressure fluctuations in time due to the energy in the $\mathrm{j}^{\text {th }}$ band by a single harmonic oscillation with frequency $\omega_{j}$ (center frequency of the band), i.e.,

$$
p_{j}(t)=\left[2 S\left(\omega_{j}\right) \Delta \omega_{j}\right]^{\frac{1}{2}} \cos \left(\omega_{j} t+\chi_{j}\right)
$$

where $\chi_{j}$ is a random number. It is easy to show that the energy associated with pressure oscillation given by Eq. (A9) is the same as $E_{j}$, i.e.,

$$
\overline{p_{j}^{2}(t)}=2 S\left(\omega_{j}\right) \Delta \omega_{j} \overline{\cos ^{2}\left(\omega_{j} t+\chi_{j}\right)}=S_{j} \Delta \omega_{j}
$$

Combining all the pressure fluctuations of all the spectral bands, the resulting pressure is obtained by summing over all the contributions given by Eq. (A9). This leads to the following equation for the random pressure fluctuations in time, 


$$
p(t)=\sum_{j=1}^{N}\left[2 S\left(\omega_{j}\right) \Delta \omega_{j}\right]^{\frac{1}{2}} \cos \left(\omega_{j} t+\chi_{j}\right)
$$

Equation (A10) is the energy conserving discretization of the given spectrum $S(\omega)$.

\section{References}

${ }^{1}$ Motsinger, R.E., and Kraft, R.E., "Design and Performance of Duct Acoustic Treatment," in Aeroacoutics of Flight Vehicles: Theory and Practice, ed. H.H. Hubbard, NASA RP 1258, Aug. 1991, Vol. 2, chapter 14, pp.165206.

${ }^{2}$ Zorumski, W.E., and Tester, B.J., "Prediction of the Acoustic Impedance of Duct Liners," NASA TM X$73951,1976$.

${ }^{3}$ Ingard, U., "On the Theory and Design of Acoustic Resonators," Journal of the Acoustical Society of America, Vol. 25, No. 6, 1955, pp. 1037-1061.

${ }^{4}$ Kooi, J.W., and Sarin, S.L., "An Experimental Study of the Acoustic Impedance of Helmholtz Resonator Arrays under Turbulent Boundary," AIAA Paper 81-1998, Oct. 1981.

${ }^{5}$ Rice, E.J., "A Model for the Pressure Excitation Spectrum and Acoustic Impedance of Sound Absorbers in the Presence of Grazing Flow," AIAA Paper 73-995, Oct. 1973.

${ }^{6}$ Hersh, A.S., and Walker, B., "The Acoustic Behavior of Helmholtz Resonators Exposed to High Speed Grazing Flows," AIAA Paper 76-536, 1976.

${ }^{7}$ Seybert, A.F., and Parrott, T.L., "Impedance Measurement Using a Two Microphone, Random Excitation Method," NASA TM78785, 1978.

${ }^{8}$ Dean, P.D., "An In-situ Method of Wall Acoustic Impedance Measurement in Flow Ducts," Journal of Sound and Vibration, Vol. 34, No. 1, 1974, pp. 97-130.

${ }^{9}$ Ingard, U., and Labate, S., "Acoustic Circulation Effects and the Nonlinear Impedance of Orifice," Journal of the Acoustical Society of America, Vol 22, 1950, 211-219.

${ }^{10}$ Hersh, A.S., and Walker, B., "Acoustic Behavior of Helmholtz Resonators-Part 1, Nonlinear Model," AIAA Paper 1995-0078, 1995.

${ }^{11}$ Jones, M.G., and Parrott, T.L., "Evaluation of a Multi-Point Method for Determining Acoustic Impedance," Journal of Mechanical Systems and Signal Processing, Vol. 3, No. 1, 1989, pp. 15-35.

${ }^{12}$ Tam, C.K.W., and Kurbatskii, K.A., "Microfluid Dynamics and Acoustics of Resonant Liners," AIAA Journal, Vol. 38, No. 8, 2000, pp. 1331-1339.

${ }^{13}$ Tam, C.K.W., and Kurbatskii, K.A., "Multi-size-mesh Multi-time-step Dispersion-Relation-Preserving scheme for Muliple-Scales Aeroacoustics Problems," International Journal of Computational Fluid Dynamics, Vol. 17, 2003, pp. 119-132.

${ }^{14}$ Melling, T.H., "The Acoustic Impedance of Perforates at Medium and High Pressure Levels," Journal of Sound and Vibration, Vol. 29, 1973, pp.1-65.

${ }^{15}$ Tam, C.K.W., Kurbatskii, K.A., Ahuja, K.K., and Gaeta, R.J.Jr., "A Numerical and Experimental Investigation of the Dissipation Mechanisms of Resonant Acoustic Liners," Journal of Sound and Vibration, Vol. 245, No. 3, 2001, pp. 545-557.

${ }^{16}$ Tam, C.K.W., Ju, H., Jones, M.G., Watson, W.R., and Parrott, T.L., "A Computational and Experimental Study of Slit Resonators," Journal of Sound and Vibration, Vol. 284, 2005, pp. 947-984.

${ }^{17}$ Sivian, L.J., "Acoustic Impedance of Small Orifices," Journal of Acoustical Society of America, Vol. 7, 1935.

${ }^{18}$ White, F.M., Viscous Fluid Flow, $2^{\text {nd }}$ ed. McGraw Hill, New York, 1991 (Chapter 3).

${ }^{19}$ Tam, C.K.W., and Webb, J.C., "Dispersion-Relation-Preserving Finite Difference Scheme for Computational Acoustics," Journal of Computational Physics, Vol. 107, 1993, pp. 262-281. 